\title{
Study on Factors Related to Home Quarantine and Smoking Behavior for People Trying to Quit Smoking in Beijing Communities during the Epidemic of COVID-19 Period and its Inspiration to Tobacco Control Policies
}

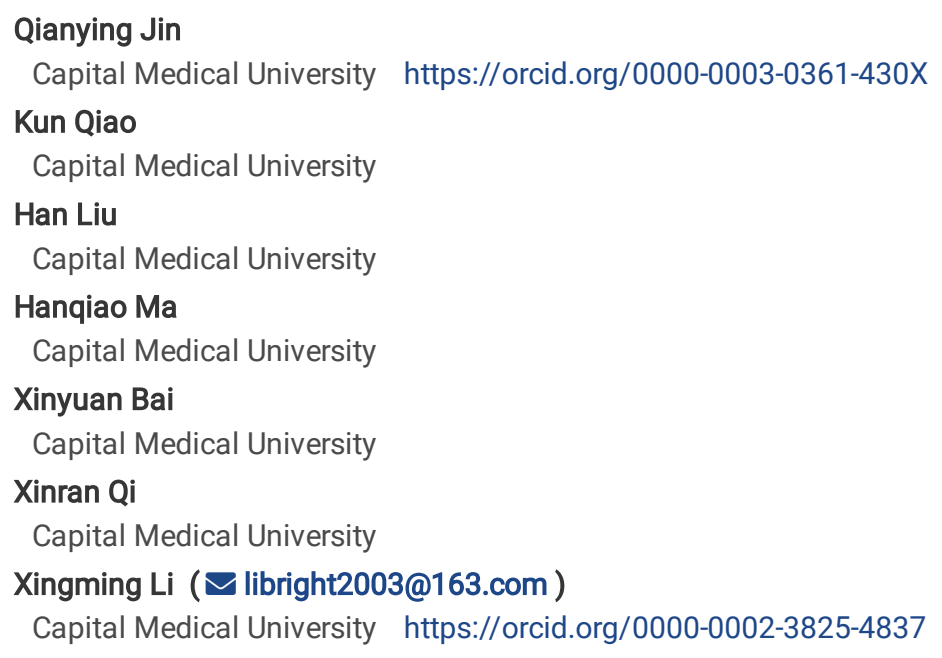

\section{Research}

Keywords: COVID-19, People Trying to Quit smoking, Home Quarantine, Tobacco Control, Propensity Score Matching

Posted Date: July 14th, 2021

DOI: https://doi.org/10.21203/rs.3.rs-678155/v1

License: (c) (i) This work is licensed under a Creative Commons Attribution 4.0 International License. Read Full License 


\section{Abstract}

Background: To understand the quit smoking status of people trying to quit smoking in Beijing communities during the epidemic of COVID-19 period, analyze their changes in smoking behavior, relapse thought, nicotine dependence, willingness to quit smoking compared with before the epidemic, and correlation with home stay time, change in psychological mood and smoking status during independent home quarantine so as to provide reference for tobacco control and optimization of quitting smoking guidance service during normalization of epidemic prevention and control.

Methods: To interview 388 people who are trying to quit smoking and involved in quitting smoking intervention service by one-to-one face interview before outbreak and one-to-one telephone survey during the epidemic period. The survey covers daily home stay time, psychological mood, tobacco use, nicotine dependence, and willingness to quit smoking. Adopt 1:2 propensity score matching, and take whether to consume quit smoking drugs and whether to participate in regular follow-ups as the matching conditions to control confounder. After matching, the $c^{2}$ test, $t$ test, and rank sum test were used to compare correlative factors such as smoking behavior change, relapse thought, nicotine dependence, and change in willingness to quit smoking between people who have quit and those who have not quit.

Results: A total of 348 cases were successfully matched, including 137 who have quit smoking and 211 who have not quit smoking. The difference in two groups of baseline data after matching was no statistically significant $(P>0.05)$. During the epidemic period, the quit smoking rate $(39.9 \%)$ was higher than that $(25.0 \%)$ before the outbreak. The average daily smoking $(7.10 \pm 8.473)$ was lower than that $(7.77 \pm 8.405)$ before the outbreak. The score of Fagerstrom Test of Nicotine Dependence (1.91 \pm 2.449$)$ was lower than that (4.35 \pm 2.518$)$ before the outbreak; the score of the assessment scale of willingness to quit smoking (38.17 \pm 12.377$)$ was higher than that (19.78 \pm 9.103$)$ before the outbreak; the smoking change of non-smokers before and after outbreak was related to distress, calm psychological mood, relief of sorrow and boredom, smoking addiction and habit $(P<0.05)$; nicotine dependence was related to the psychological mood of distress $(P<0.05)$, and the willingness to quit smoking was related to the daily home stay time $(\mathrm{P}<0.05)$; For those who had quit smoking successfully, the smoking change was related to the psychological mood of distress, relief of sorrow and boredom, alleviation of stress, smoking addiction and habit ( $P<0.05)$; relapse thought was related to the psychological mood of distress, relief of sorrow and boredom, recreation and alleviation of stress $(P<0.05)$; nicotine dependence was related to the daily home stay time, calm psychological mood and smoking addition $(\mathrm{P}<0.05)$, and the willingness to quit smoking related to recreation and smoking addition $(\mathrm{P}<0.05)$.

Conclusions: During the epidemic period, people who tried to quit smoking reduced their smoking, nicotine dependence, and their willingness to quit smoking increased. During normalization of epidemic prevention and control, social activities decrease and home stay time increased, which created favorable conditions for quitting smoking and tobacco control. However, negative emotions during home stay, relief of sorrow and boredom and smoking addition may restore smoking behavior and relapse thought. It is recommended that family members provide more support and encouragement to smoke quitters, and supervise and help them reduce smoking. During normalization of epidemic prevention and control, the quit smoking service center should alleviate negative emotion of quitters and break connection between existing smoking habit and negative emotion and environmental temptation.

\section{Background}

The pandemic of COVID-19 broke out in December 2019 and spread out quickly. In order to control the disease, the Chinese government and the Beijing municipal government have called on the public for home quarantine at their own volition. According to relevant findings, mental health of people during home quarantine has significantly decreased. Such factors as disruption on the original life order and confusion may lead to psychological stress, discomfort, distress and rising stress of quarantined people. However, appropriate cognition and behavior adjustment can alleviate psychological anxiety ${ }^{[1-2]}$.

Before the outbreak, our research team had carried out quit smoking interventions in some Beijing communities ${ }^{[3-4]}$. 685 tobacco-dependent patients in Beijing communities were trying to quit smoking. However, quitting smoking is complex and closely related to social and psychological factors. The success of quit smoking is related to many factors such as different smoking status, motivation of quitting smoking, and temptations during quitting smoking ${ }^{[5]}$. The several-month home quarantine and the psychological mood change during quarantine may affect quit smoking status, willingness to quit smoking and psychological mood. Therefore, the smoking behavior of the quitters may change. However, there is currently absence of research on the epidemic impact on smoking quitters. In order to probe into the changes in quitting smoking behaviors and related factors of people trying to quit smoking during the epidemic period, the Propensity Score Matching (PSM) is planned to be adopted to control the impact of related confounders and analyze correlation between changes in smoking behavior, nicotine dependence, and willingness to quit smoking and home stay time and psychological mood of people trying to quit smoking in Beijing communities during The epidemic of COVID-19 period (February-April, 2020), thus providing reference for optimization of quitting smoking service and bettering of guidance during normalization of epidemic prevention and control.

\section{Subject And Methods}




\section{Subjects}

This was a key research and development program of the Ministry of Science and Technology in 2017, and the subject was the tobaccodependent patients who were successively enrolled for quit smoking intervention from December 2018 to December 2019. Quit smoking campaign was conducted in 19 Beijing communities to recruit and screen smokers willing to quit. The investigators and doctors in quit smoking outpatient conducted one-to-one face-to-face questionnaire survey, carbon monoxide blowing test, and pulmonary function test on included smokers. Based on the test results, the doctor formulated a specific quit smoking program for each subject, including whether to consume quit smoking drugs, daily dose, daily smoking reduction schedule, and precautions. Throughout the research, the subjects would receive a three-month quit smoking intervention and six-month follow-up. From March 2020 to April 2020, the research team recruited and trained telephone investigators. Such information as smoking status and willingness to quit smoking during epidemic period were updated timely by one-to-one telephone follow-up.

Inclusion criteria: $₫ 18$ years old and above; \permanent residents in Beijing community; $₫$ smokers, including regular smokers (smoking more than one cigarette per day, continuous or cumulative for 6 months ${ }^{[6]}$ ), occasional smokers (smoking cigarettes more than 4 times a week, but less than one cigarette per day on average); $\otimes$ communicate fluently and be willing to get involved in the survey. "quit smoking" refers to 7-day quit rate. The subjects reported that they quitted smoking $\geq 7 \mathrm{~d}^{[7]}$. "No quit" refers to those who still smoke during survey, including those who had quit smoking but relapsed. In the final telephone survey, 388 people trying to quit smoking were successfully followed up. 177 people said they rejected the telephone survey, and 120 people failed to be connected after the investigator who tried to contact them by phone for many times.

\section{Research Contents}

Investigation contents before outbreak include: (1) Demographic information, including information such as age, gender, marital status, and education level; (2) Tobacco use, including daily smoking and smoking time; (3) Fagerstrom Test of Nicotine Dependence (FTND); (4) Assessment Scale of Willingness to Quit Smoking.

Telephone follow-up contents during the epidemic period include: (1) Daily home stay time in the past two weeks. The answers include: almost all day, $80-90 \%$ of the time, $50-80 \%$ of the time, and less than $50 \%$ of the time; (2) Words to describe psychological mood during epidemic period? The answers include: suspect, anxiety, panic, distress, calmness, and the same as usual. "Calmness" refers to the subject's ability to think rationally about the epidemic, and "the same as usual" refers to the indifferent attitude; (3) Smoking behavior of the people trying to quit smoking, including daily smoking, resumption of smoking thought because of epidemic (it refers to relapse in case of already having quit); (4) Fagerstrom Test of Nicotine Dependence (FTND); (5) If smoking continues, the main reason for the smoking behavior, answers include: refreshing, relief of sorrow and boredom, recreation, socializing, intimation, curiosity, influence by the elderly, alleviation of stress, smoking addiction; (6) Assessment Scale of Willingness to Quit Smoking.

Fagerstrom Test of Nicotine Dependence (FTND) includes 6 items, and each item is scored by $0-3$ points, representing the dependence in ascending order. Nicotine dependence is judged based on 6 items, including 3 degrees of dependence: mild: 0-3 points; moderate: 4-6 points; severe: 7 points or more. The Scale was first compiled by the scholar Fagerstrom, et al. in 1989 and revised by Heatherton, et al. in 1991. The Chinese version was translated and introduced by Huang CL, et al. in 2006 and its structural validity was preliminarily verified. The Cronbach's a coefficient of the Chinese version of the Scale is $0.658^{[8]}$, indicating that its internal consistency reliability is acceptable.

The assessment scale of willingness to quit smoking was designed by the research team to assess the subjects' willingness to quit smoking. The Scale contains ten items, subject to Likert 5-point scoring standard, "very non-conforming" rated as 1 point, "relatively non-conforming" rated as 2 points, "general" rated as 3 points, "relatively conforming" rated as 4 points, and "very conforming" is rated as 5 points. The higher the score, the stronger the willingness to quit smoking. By calculating the total score of each subject, it was divided into very low willingness to quit smoking (total score $<10$ points), relatively low (10 points $\leq$ total score $<19$ points), general (20 points $\leq$ total score $<29$ points), relatively high (30 points $\leq$ total score $<39$ points), very high (total score $\geq 40$ points). The Cronbach's aacoefficient was tested to be 0.805 , the $\mathrm{KMO}$ value 0.895 , and the Bartlett value is $872.096(\mathrm{P}<0.001)$. After the maximum variance rotation, the four factors are extracted and the cumulative explanation is $86 \%$, indicating that the scale has a higher reliability ${ }^{[3-4]}$.

\section{Statistical Analysis}

IBM SPSS Statistics 22.0 and R 2.15 .3 software were used for propensity score matching and statistical analysis. Propensity Score Matching (PSM) is a statistical method commonly used to process observational research data. This method is used to mitigate influence of data bias and confounder for more reasonable comparison on the trial group and control group. On this study, subjects were divided into two groups, those who had quit smoking and those who had not quit smoking. Nearest neighbor matching (NNM) was used. Based on the propensity score of the subjects in the quitter group, seek those among non-quitters nearest to the score. Set the caliper value of the propensity score as 0.1 , perform a

Page $3 / 19$ 
1:2 pairing, and conduct subsequent analysis by the matching result. The matching variable is whether quit smoking is successful. The matching conditions include whether to consume quit smoking drug, or receive regular follow-up. Finally, 137 cases of quitters and 211 cases of non-

quitters were successfully matched, with a total of 348 cases.

Besides, a descriptive analysis method was adopted to conduct statistics of the overall distribution of people trying to quit smoking during the epidemic period, such as home stay time, psychological mood, smoking behavior, and willingness to quit smoking. The measurement data are expressed as $\llbracket x \pm s$, and the inter-group comparison uses $t$ test. The count data are expressed by percentage (\%). $\chi^{2}$ test and the rank sum test were used to conduct correlation analysis of home stay time, psychological mood, smoking reason of people trying to quit smoking and smoking change and willingness to quit smoking before and after COVID-19. $\mathrm{P}<0.05$ means that the difference was statistically significant.

\section{Analysis Thought}

According to research findings of the psychology of people during COVID-19 home quarantine, long-term home quarantine can lead to a series of psychological stress reactions such as loneliness, fear, and anxiety ${ }^{[9]}$. After surveying community residents, Yang Liquan ${ }^{[10]}$, et al. found that most residents have varying degrees of fear and distress. Meanwhile, home quarantine changed the daily lifestyle of people. Due to extended leisure time, change of life cycle, narrow living space, singular lifestyle and less socializing with the outside world, people are prone to negative mood ${ }^{[11]}$. Studies have shown that mood is related to smoking behavior. Smokers were not aware of the mechanism of nicotine addiction and believed that tobacco can lift people up. However, the effect of smoking may be magnified when there is negative mood ${ }^{[4,12]}$. For the smokers trying to quit smoking in this study, home quarantine during the epidemic period made them accept life events and environmental stressors, which might also produce negative psychological mood. Psychosocial social factor is an important factor to influence nicotine dependence, as well as smokers' willingness to quit smoking and smoking status. The success of quit smoking is affected by many factors, including different smoking status, motivation of quitting smoking, whether appropriate techniques and methods are available, and temptations during quit smoking period [5]. After COVID-19 outbreak, the results of the telephone follow-up showed that the average daily smoking of 165 people trying to quit smoking changed over that before outbreak, accounting for $42.5 \%$ of people receiving telephone survey. And the difference of distribution of smoking change in the two groups of subjects was statistically significant $(P<0.05)$, see Table 1 . Further comparison of the daily smoking, nicotine dependence, and evaluation of the willingness to quit smoking of people trying to quit smoking showed that the distribution difference compared with that before outbreak was statistically significant (all $P<0.05$ ), see Table 2 . According to findings, during epidemic period, the average daily smoking of the people trying to quit smoking decreased slightly, the quit smoking rate increased significantly, nicotine dependence decreased somewhat, and the willingness to quit smoking increased significantly than before the outbreak. It showed that the smoking behavior of subjects changed, and changed for better than before the outbreak.

Table 1

Comparison of changes in daily average smoking between the two groups of people trying to quit smoking before and after The epidemic of COVID-19 period [n(\%)]

\begin{tabular}{|c|c|c|c|c|}
\hline \multirow[t]{2}{*}{ Smoking quantity change } & \multicolumn{2}{|c|}{ Have you successfully quit smoking? } & \multirow[t]{2}{*}{$Z$} & \multirow[t]{2}{*}{$p$} \\
\hline & No & Yes & & \\
\hline$-35 \sim$ & $6(2.6)$ & $7(4.5)$ & -2.366 & 0.018 \\
\hline$-10 \sim$ & $41(17.6)$ & $32(20.6)$ & & \\
\hline $0 \sim$ & $129(55.4)$ & $94(60.6)$ & & \\
\hline $1 \sim$ & $50(21.5)$ & $22(14.2)$ & & \\
\hline $10 \sim$ & $7(3.0)$ & $0(0.0)$ & & \\
\hline Total & 233(100.0) & 155(100.0) & & \\
\hline
\end{tabular}

Table 2 Comparison of smoking status, nicotine dependence and willingness to quit smoking among people trying to quit smoking before and after the epidemic of COVID-19 period [ $\mathrm{x} \pm \mathrm{s}$ ]

\begin{tabular}{|c|c|c|c|c|c|}
\hline Index & $\mathrm{n}$ & Mean \pm standard deviation & Quit smoking rate (\%) & $t$ & $p$ \\
\hline Average daily smoking before outbreak & 388 & $7.77 \pm 8.405$ & 25.0 & 3.029 & 0.003 \\
\hline Average daily smoking during epidemic period & 388 & $7.10 \pm 8.473$ & 39.9 & & \\
\hline Nicotine dependence before outbreak & 388 & $4.35 \pm 2.518$ & - & 18.988 & $<0.001$ \\
\hline Nicotine dependence during epidemic period & 388 & $1.91 \pm 2.449$ & - & & \\
\hline Willingness to quit smoking before outbreak & 388 & $19.78 \pm 9.103$ & - & -22.552 & $<0.001$ \\
\hline Willingness to quit smoking during epidemic period & 388 & $38.17 \pm 12.377$ & - & & \\
\hline
\end{tabular}


However, the subjects in this study had received three-month quit smoking services and six-month follow-up, which overlapped with the epidemic period. And again, use of quit smoking drugs and regular follow-ups might also was related to change in smoking behavior of people trying to quit smoking, see Fig. 1. In order to control influence of confounders, the main content of quit smoking intervention was planned to be used as the matching conditions for propensity score matching. After matching, relevant factors could be controlled. And again, further analysis of relevant factors for changes in smoking behavior of people trying to quit smoking during epidemic period would be conducted to define correlation between people's home stay time, psychological mood, smoking status and their behavior change.

\section{Ethics Committee Approval}

This study has been approved by the Ethics Committee of the Capital Medical University (Z2019SY007), and an informed consent form signed with the subject before study. In addition, this study has been registered in the official website of Chinese Clinical Trial Registry

(Chictr1900024991).

\section{Results}

\section{Comparison of two groups of baseline data}

There were 388 subjects before matching. Among people trying to quit smoking during the telephone follow-up, 155 people successfully quit smoking while 233 people continued smoking. Subjects were mostly male (349 people, 89.9\%), and subjects were at 60 or above highest (163, $42.0 \%)$; in terms of marital status, most subjects were married $(342,88.1 \%)$; the education focused on senior high school graduates (212,54.6\%). Before matching, the difference of two groups of subjects was statistically significant in terms of age, drug use and regular follow-up ratio.

After matching, 348 subjects were included, including 137 quitters and 211 non-quitters. There were 315 males, accounting for $90.5 \%$; 143 people at 60 or above, accounting for $41.1 \%$; 308 subjects were married, accounting for $88.5 \%$; 198 subjects were senior high school graduates accounting for $56.9 \%$. Difference of two groups of baseline data after matching was not statistically significant $(P>0.05)$, see Table 3 .

Table 3 Subject baseline data and PSM matching results during the epidemic of COVID-19 period [n (\%)] 


\begin{tabular}{|c|c|c|c|c|c|c|c|c|}
\hline \multirow[t]{2}{*}{ Variable } & \multicolumn{4}{|c|}{ Before matching $(n=388)$} & \multicolumn{4}{|c|}{ After matching $(\mathrm{n}=348)$} \\
\hline & $\begin{array}{l}\text { No } \\
\text { quit(n=233) }\end{array}$ & Quit( $n=155)$ & $t / \boldsymbol{X}^{2}$ & $p$ & $\begin{array}{l}\text { No } \\
\text { quit( }(n=211)\end{array}$ & Quit(n=137) & $t / \chi^{2}$ & $p$ \\
\hline Male & 212(91.0) & 137(88.4) & 0.438 & 0.508 & 193(91.5) & 122(89.1) & 0.319 & 0.572 \\
\hline$\left[\mathrm{M}\left(\mathrm{P}_{25}, \mathrm{P}_{75}\right)\right]$ Age & $56(40,72)$ & $61(46,76)$ & -1.983 & 0.048 & $56(40,72)$ & $59(44,74)$ & -1.535 & 0.126 \\
\hline \multicolumn{9}{|l|}{ Marital status } \\
\hline Single & $18(7.7)$ & $4(2.6)$ & \multirow[t]{5}{*}{8.552} & \multirow[t]{5}{*}{0.073} & 15(7.1) & $4(2.9)$ & \multirow[t]{5}{*}{6.125} & \multirow[t]{5}{*}{0.190} \\
\hline Married & 197(84.5) & 145(93.5) & & & $180(85.3)$ & 128(93.4) & & \\
\hline Separated & $2(0.9)$ & $0(0.0)$ & & & $2(0.9)$ & $0(0.0)$ & & \\
\hline Divorced & $11(4.7)$ & $3(1.9)$ & & & $10(4.7)$ & $3(2.2)$ & & \\
\hline Widowed & $5(2.1)$ & $3(1.9)$ & & & $4(1.9)$ & $2(1.5)$ & & \\
\hline \multicolumn{9}{|l|}{ Education level } \\
\hline Primary school and below & $14(6.0)$ & $9(5.8)$ & \multirow[t]{3}{*}{1.088} & \multirow[t]{3}{*}{0.581} & $11(5.2)$ & $6(4.4)$ & \multirow[t]{3}{*}{1.639} & \multirow[t]{3}{*}{0.441} \\
\hline Senior high school & 132(56.7) & $80(51.6)$ & & & $125(59.2)$ & 73(53.3) & & \\
\hline College degree and above & 87(37.3) & $66(42.6)$ & & & $75(35.5)$ & $58(42.3)$ & & \\
\hline \multicolumn{9}{|c|}{$\begin{array}{l}\text { Whether to consumer quit smoking } \\
\text { drug }\end{array}$} \\
\hline No & $40(17.2)$ & $58(37.4)$ & \multirow[t]{2}{*}{19.164} & \multirow[t]{2}{*}{$<0.001$} & $40(19.0)$ & $40(29.2)$ & \multirow[t]{2}{*}{4.358} & \multirow[t]{2}{*}{0.177} \\
\hline Yes & 193(82.8) & $97(62.6)$ & & & $171(81.0)$ & $97(70.8)$ & & \\
\hline \multicolumn{9}{|c|}{ Whether to receive regular follow-up } \\
\hline No & $100(42.9)$ & $50(32.3)$ & \multirow[t]{2}{*}{4.022} & \multirow[t]{2}{*}{0.045} & $78(37.0)$ & $46(33.6)$ & \multirow[t]{2}{*}{0.282} & \multirow[t]{2}{*}{0.596} \\
\hline Yes & $133(57.1)$ & $105(67.7)$ & & & 133(63.0) & $91(66.4)$ & & \\
\hline
\end{tabular}

\section{Distribution of home stay time, psychological mood, and smoking behavior during epidemic period}

During epidemic period, $64.2 \%$ of subjects said that they stayed at home almost all day. $80.9 \%$ of subjects said that their psychological mood during epidemic period was the same as usual, and $46.9 \%$ said that they remained calm and could rationally view the disease. However, 117 subjects also said that they had psychological emotions of anxiety, distress, panic, and suspicion. 11.3\% said that they wanted to smoke again during epidemic period. See Table 4. During epidemic period, the top three reasons that drove people trying to quit smoking to continue smoking were smoking addiction, recreation, and relief of sorrow and boredom. This might be related to the long home stay time during epidemic period. 


\begin{tabular}{|c|c|c|c|c|c|c|c|c|c|}
\hline \multirow[t]{2}{*}{ Questions } & \multirow[t]{2}{*}{ Indicators } & \multicolumn{4}{|c|}{ Before matching $(n=388)$} & \multicolumn{4}{|c|}{ After matching $(n=348)$} \\
\hline & & $\begin{array}{l}\text { Persistent } \\
\text { Smokers } \\
(\mathrm{n}=233)\end{array}$ & $\begin{array}{l}\text { Ex- } \\
\text { smokers } \\
(n=155)\end{array}$ & $t / \chi^{2}$ & $p$ & $\begin{array}{l}\text { Persistent } \\
\text { Smokers } \\
(n=211)\end{array}$ & $\begin{array}{l}\text { Ex- } \\
\text { smokers } \\
(n=137)\end{array}$ & $t / \chi^{2}$ & $p$ \\
\hline \multirow[t]{4}{*}{$\begin{array}{l}\text { The length of staying at home } \\
\text { per day in the past two weeks }\end{array}$} & $\begin{array}{l}\text { Almost all } \\
\text { day }\end{array}$ & 149(63.9) & $100(64.5)$ & \multirow[t]{4}{*}{1.125} & \multirow[t]{4}{*}{0.771} & $135(64.0)$ & $87(63.5)$ & \multirow[t]{4}{*}{1.643} & \multirow[t]{4}{*}{0.650} \\
\hline & $\begin{array}{l}80 \%-90 \% \text { of } \\
\text { the day }\end{array}$ & $22(9.4)$ & 19(12.3) & & & $18(8.5)$ & $17(12.4)$ & & \\
\hline & $\begin{array}{l}50 \%-80 \% \text { of } \\
\text { the day }\end{array}$ & $31(13.3)$ & 18(11.6) & & & $27(12.8)$ & 16(11.7) & & \\
\hline & $\begin{array}{l}\text { Less than } \\
50 \% \text { of the } \\
\text { day }\end{array}$ & $31(13.3)$ & 18(11.6) & & & $31(14.7)$ & $17(12.4)$ & & \\
\hline \multirow{6}{*}{$\begin{array}{l}\text { Which of the following words } \\
\text { can describe your recent } \\
\text { psychological state during the } \\
\text { epidemic (multiple choice) }\end{array}$} & Suspected & $5(2.1)$ & $0(0.0)$ & 1.894 & 0.169 & $4(1.9)$ & $0(0.0)$ & 1.224 & 0.269 \\
\hline & Worried & $48(20.6)$ & 19(12.3) & 4.535 & 0.033 & $45(21.3)$ & 15(10.9) & 6.270 & 0.012 \\
\hline & Panic & $11(4.7)$ & $7(4.5)$ & 0.009 & 0.925 & $10(4.7)$ & $6(4.4)$ & 0.025 & 0.876 \\
\hline & Depressed & 19(8.2) & $8(5.2)$ & 1.288 & 0.256 & $18(8.5)$ & $8(5.8)$ & 0.870 & 0.351 \\
\hline & Calm & $96(41.2)$ & $86(55.5)$ & 7.624 & 0.006 & $88(41.7)$ & $77(56.2)$ & 7.003 & 0.008 \\
\hline & $\begin{array}{l}\text { Almost as } \\
\text { usual }\end{array}$ & 181(77.7) & 133(85.8) & 3.980 & 0.046 & 164(77.7) & 119(86.9) & 4.565 & 0.033 \\
\hline \multirow{2}{*}{$\begin{array}{l}\text { Although you have quit } \\
\text { smoking, } \\
\text { do you want to smoke } \\
\text { because of the epidemic } \\
\text { (namely relapse) }\end{array}$} & No & 206(88.4) & 138(89.0) & \multirow[t]{2}{*}{0.036} & \multirow[t]{2}{*}{0.850} & 185(87.7) & 121(88.3) & \multirow[t]{2}{*}{0.032} & \multirow[t]{2}{*}{0.857} \\
\hline & Yes & 27(11.6) & 17(11.0) & & & 26(12.3) & 16(11.7) & & \\
\hline \multirow{7}{*}{$\begin{array}{l}\text { If you still smoke now, the main } \\
\text { reason is } \\
\text { (multiple choice) }\end{array}$} & Refreshing & $4(1.7)$ & $0(0.0)$ & 2.689 & 0.101 & $4(1.9)$ & $0(0.0)$ & 1.224 & 0.269 \\
\hline & $\begin{array}{l}\text { Dispelling } \\
\text { depression } \\
\text { and boredom }\end{array}$ & $50(21.5)$ & $6(3.9)$ & 23.315 & $<.001$ & $46(21.8)$ & $5(3.6)$ & 21.881 & $<.001$ \\
\hline & Entertaining & $55(23.6)$ & $4(2.6)$ & 31.910 & $<.001$ & $52(24.6)$ & $4(2.9)$ & 29.035 & $<.001$ \\
\hline & $\begin{array}{l}\text { Social and } \\
\text { business } \\
\text { activities }\end{array}$ & 17(7.3) & $1(0.6)$ & 9.307 & 0.002 & $17(8.1)$ & $1(0.7)$ & 9.092 & 0.003 \\
\hline & $\begin{array}{l}\text { Imitating out } \\
\text { of curiosity }\end{array}$ & $2(0.9)$ & $0(0.0)$ & 2.047 & 0.153 & $2(0.9)$ & $0(0.0)$ & 2.009 & 0.156 \\
\hline & $\begin{array}{l}\text { Reliving } \\
\text { pressure }\end{array}$ & 18(7.7) & $1(0.6)$ & 10.019 & 0.002 & $15(7.1)$ & $1(0.7)$ & 7.706 & 0.006 \\
\hline & $\begin{array}{l}\text { Other } \\
\text { reasons } \\
\text { (addiction to } \\
\text { smoking, } \\
\text { habits, etc.) }\end{array}$ & $81(34.8)$ & $3(1.9)$ & 59.138 & $<.001$ & 72(34.1) & $3(2.2)$ & 50.101 & $<.001$ \\
\hline \multirow{3}{*}{$\begin{array}{l}\text { Degree of nicotine dependence } \\
\text { before the epidemic }\end{array}$} & Light & $86(36.9)$ & 105(67.7) & \multirow[t]{3}{*}{-5.822} & \multirow{3}{*}{$\begin{array}{l}< \\
0.001\end{array}$} & $82(38.9)$ & $91(66.4)$ & \multirow[t]{3}{*}{-4.893} & \multirow{3}{*}{$<.001$} \\
\hline & Moderate & $116(49.8)$ & $41(26.5)$ & & & 101(47.9) & $37(27.0)$ & & \\
\hline & Heavy & $31(13.3)$ & $9(5.8)$ & & & 28(13.3) & $9(6.6)$ & & \\
\hline \multirow{3}{*}{$\begin{array}{l}\text { Degree of nicotine dependence } \\
\text { during the epidemic }\end{array}$} & Light & 175(75.1) & 148(95.5) & \multirow[t]{3}{*}{-5.280} & \multirow{3}{*}{$<.001$} & 159(75.4) & $130(94.9)$ & \multirow[t]{3}{*}{-4.752} & \multirow{3}{*}{$<.001$} \\
\hline & Moderate & $52(22.3)$ & $7(4.5)$ & & & $49(23.2)$ & $7(5.1)$ & & \\
\hline & Heavy & $6(2.6)$ & $0(0.0)$ & & & $3(1.4)$ & $0(0.0)$ & & \\
\hline
\end{tabular}




\begin{tabular}{|c|c|c|c|c|c|c|c|c|c|}
\hline \multirow{5}{*}{$\begin{array}{l}\text { Assessment of willingness to } \\
\text { quit smoking before the } \\
\text { epidemic }\end{array}$} & Very low & $33(14.2)$ & $25(16.1)$ & \multirow[t]{5}{*}{-3.444} & \multirow[t]{5}{*}{0.001} & 29(13.7) & 21(15.3) & \multirow[t]{5}{*}{-3.138} & \multirow[t]{5}{*}{0.002} \\
\hline & $\begin{array}{l}\text { Relatively } \\
\text { low }\end{array}$ & 71(30.5) & $78(50.3)$ & & & 67(31.8) & $70(51.1)$ & & \\
\hline & Moderate & $95(40.8)$ & $38(24.5)$ & & & $85(40.3)$ & $34(24.8)$ & & \\
\hline & $\begin{array}{l}\text { Relatively } \\
\text { strong }\end{array}$ & $30(12.9)$ & $13(8.4)$ & & & $26(12.3)$ & $11(8.0)$ & & \\
\hline & Very strong & $4(1.7)$ & $1(0.6)$ & & & $4(1.9)$ & $1(0.7)$ & & \\
\hline \multirow{5}{*}{$\begin{array}{l}\text { Assessment of willingness to } \\
\text { quit smoking during the } \\
\text { epidemic }\end{array}$} & Very low & 13(5.6) & $3(1.9)$ & \multirow[t]{5}{*}{-0.639} & \multirow[t]{5}{*}{0.523} & $12(5.7)$ & $3(2.2)$ & \multirow[t]{5}{*}{-0.005} & \multirow[t]{5}{*}{0.996} \\
\hline & $\begin{array}{l}\text { Relatively } \\
\text { low }\end{array}$ & $13(5.6)$ & $5(3.2)$ & & & $13(6.2)$ & $4(2.9)$ & & \\
\hline & Moderate & $22(9.4)$ & $29(18.7)$ & & & $22(10.4)$ & $24(17.5)$ & & \\
\hline & $\begin{array}{l}\text { Relatively } \\
\text { strong }\end{array}$ & $60(25.8)$ & $42(27.1)$ & & & $54(25.6)$ & $37(27.0)$ & & \\
\hline & Very strong & 125(53.6) & $76(49.0)$ & & & $110(52.1)$ & $69(50.4)$ & & \\
\hline
\end{tabular}

After matching, the degree of nicotine dependence before the epidemic and during the epidemic, of the assessment of willingness to quit smoking before the epidemic between two groups was statistically significant $(P<0.05)$, as well as in the form of numerical variables $(T h e$ mean standard deviations of their three indicators of Persistent Smokers and Ex-smokers are4.92 $\pm 2.357,3.48 \pm 2.451 ; 2.69 \pm 2.320,0.61 \pm 1.969 ; 20.98 \pm 9.200,17.88 \pm 8.456$, respectively) See Table 4. and Table 5. Compared with before outbreak, the nicotine dependence of quitters and non-quitters both decreased, and the willingness to quit smoking increased significantly.

Table 5 Comparison of Degree of Nicotine Dependence and Willingness to Quit Smoking of Subjects before and during the epidemic of COVID-19 [ $\overline{\mathrm{x}} \pm \mathrm{S}$ ]

\begin{tabular}{|c|c|c|c|c|c|c|c|c|}
\hline \multirow[t]{2}{*}{ Indicators } & \multicolumn{4}{|c|}{ Before Matching $(n=388)$} & \multicolumn{4}{|c|}{ After Matching ( $n=348)$} \\
\hline & $\begin{array}{l}\text { Persistent } \\
\text { Smokers }(n= \\
\text { 233) }\end{array}$ & $\begin{array}{l}\text { Ex-smokers } \\
(n=155)\end{array}$ & $t$ & $p$ & $\begin{array}{l}\text { Persistent } \\
\text { Smokers }(n= \\
\text { 211) }\end{array}$ & $\begin{array}{l}\text { Ex-smokers } \\
(n=137)\end{array}$ & $t$ & $p$ \\
\hline $\begin{array}{l}\text { Degree of nicotine dependence } \\
\text { before the epidemic }\end{array}$ & $5.01 \pm 2.353$ & $3.37 \pm 2.443$ & 6.636 & $<001$ & $4.92 \pm 2.357$ & $3.48 \pm 2.451$ & 5.465 & $\dot{0} 001$ \\
\hline $\begin{array}{l}\text { Degree of nicotine dependence } \\
\text { during the epidemic }\end{array}$ & $2.77 \pm 2.384$ & $0.61 \pm 1.917$ & 9.456 & $\begin{array}{l}< \\
0.001\end{array}$ & $2.69 \pm 2.320$ & $0.61 \pm 1.969$ & 8.671 & $\dot{0} 001$ \\
\hline $\begin{array}{l}\text { Assessment of willingness to } \\
\text { quit smoking before the } \\
\text { epidemic }\end{array}$ & $20.97 \pm 9.365$ & $17.98 \pm 8.410$ & 3.198 & 0.001 & $20.98 \pm 9.200$ & $17.88 \pm 8.456$ & 3.167 & 0.002 \\
\hline $\begin{array}{l}\text { Assessment of willingness to } \\
\text { quit smoking during the } \\
\text { epidemic }\end{array}$ & $38.31 \pm 12.897$ & $37.95 \pm 11.590$ & 0.275 & 0.784 & $37.71 \pm 12.995$ & $38.21 \pm 11.781$ & -0.359 & 0.720 \\
\hline
\end{tabular}

\section{Analysis of factors related to daily tobacco consumption change of the two groups}

During the epidemic, the smoking behavior of people trying to quit smoking changed, specifically speaking, the decrease or increase in daily tobacco consumption. The analysis results showed that for people who are trying to quit smoking but haven't succeeded, the change of their daily tobacco consumption is mainly related to such factors as the generation of smoking idea, depression, calmness, relieving sorrow and boredom, relieving pressure, addiction to smoking and habits. The distributional difference is of statistical significance (all $\mathrm{P}<0.05)$, see Table 6 . For test patients who have quit smoking, factors like depression, relieving sorrow and boredom, relieving pressure, addiction to smoking and habits, degree of nicotine dependence before the epidemic, and the willingness to quit before and during the epidemic are related to the changes in tobacco consumption. The distributional difference is statistically significant $(P<0.05)$. 
Table 6

Factors Related to Changes in Daily Tobacco Consumption of Subjects during the COVID-19 Outbreak [ $\overline{\mathrm{x}} \pm \mathrm{s}$ ]

\begin{tabular}{|c|c|c|c|c|c|c|c|c|c|}
\hline \multirow[t]{2}{*}{ Questions } & \multirow[t]{2}{*}{ Degree } & \multicolumn{4}{|c|}{ Persistent Smokers $(n=211)$} & \multicolumn{4}{|c|}{ Ex-smokers $(n=137)$} \\
\hline & & $\mathrm{n}$ & $\begin{array}{l}\text { Daily tobacco } \\
\text { consumption }\end{array}$ & $t$ & $p$ & $\mathrm{n}$ & $\begin{array}{l}\text { Daily } \\
\text { tobacco } \\
\text { consumption }\end{array}$ & $t$ & $p$ \\
\hline \multirow[t]{4}{*}{$\begin{array}{l}\text { The length of staying at home per day in } \\
\text { the past two weeks }\end{array}$} & $\begin{array}{l}\text { Almost all } \\
\text { day }\end{array}$ & 135 & $0.05 \pm 4.462$ & \multirow[t]{4}{*}{0.811} & \multirow[t]{4}{*}{0.418} & 87 & $-1.43 \pm 3.870$ & \multirow[t]{4}{*}{0.796} & \multirow[t]{4}{*}{0.102} \\
\hline & $\begin{array}{l}80 \%-90 \% \text { of } \\
\text { the day }\end{array}$ & 18 & $-0.44 \pm 1.503$ & & & 17 & $-1.96 \pm 4.143$ & & \\
\hline & $\begin{array}{l}50 \%-80 \% \text { of } \\
\text { the day }\end{array}$ & 27 & $-0.06 \pm 3.457$ & & & 16 & $-0.63 \pm 1.857$ & & \\
\hline & $\begin{array}{l}\text { Less than } \\
50 \% \text { of the } \\
\text { day }\end{array}$ & 31 & $-0.71 \pm 5.593$ & & & 17 & $-2.47 \pm 8.552$ & & \\
\hline \multirow{2}{*}{$\begin{array}{l}\text { Although you have quit smoking, do you } \\
\text { want to smoke because of the epidemic } \\
\text { (namely relapse) }\end{array}$} & No & 185 & $-0.41 \pm 4.319$ & \multirow[t]{2}{*}{-2.664} & \multirow[t]{2}{*}{0.008} & 121 & $-1.36 \pm 3.613$ & \multirow[t]{2}{*}{1.199} & \multirow[t]{2}{*}{0.233} \\
\hline & Yes & 26 & $1.98 \pm 4.047$ & & & 16 & $-2.81 \pm 9.020$ & & \\
\hline \multirow[t]{2}{*}{ Suspected } & No & 207 & $-0.10 \pm 4.377$ & \multirow[t]{2}{*}{0.524} & \multirow[t]{2}{*}{0.601} & 137 & $-1.53 \pm 4.551$ & \multirow[t]{2}{*}{-} & \multirow[t]{2}{*}{-} \\
\hline & Yes & 4 & $-1.25 \pm 2.500$ & & & 0 & - & & \\
\hline \multirow[t]{2}{*}{ Worried } & No & 166 & $-0.23 \pm 4.662$ & \multirow[t]{2}{*}{-0.730} & \multirow[t]{2}{*}{0.466} & 122 & $-1.51 \pm 4.558$ & \multirow[t]{2}{*}{0.132} & \multirow[t]{2}{*}{0.895} \\
\hline & Yes & 45 & $0.30 \pm 2.921$ & & & 15 & $-1.68 \pm 4.643$ & & \\
\hline \multirow[t]{2}{*}{ Panic } & No & 201 & $-0.21 \pm 4.360$ & \multirow[t]{2}{*}{-1.433} & 0.153 & 131 & $-1.56 \pm 4.628$ & -0.292 & 0.770 \\
\hline & Yes & 10 & $1.80 \pm 3.824$ & & & 6 & $-1.00 \pm 2.449$ & & \\
\hline Depressed & No & 193 & $-0.34 \pm 4.258$ & -2.475 & 0.014 & 129 & $-1.60 \pm 4.675$ & -0.740 & 0.035 \\
\hline & Yes & 18 & $2.28 \pm 4.713$ & & & 8 & $-0.38 \pm 1.061$ & & \\
\hline Calm & No & 123 & $0.41 \pm 4.634$ & 2.125 & 0.035 & 60 & $-1.22 \pm 3.854$ & 0.719 & 0.473 \\
\hline & Yes & 88 & $-0.86 \pm 3.818$ & & & 77 & $-1.78 \pm 5.039$ & & \\
\hline Almost as usual & No & 47 & $0.67 \pm 3.672$ & 1.147 & 0.158 & 18 & $-1.57 \pm 4.263$ & -0.033 & 0.973 \\
\hline & Yes & 164 & $-0.35 \pm 4.509$ & & & 119 & $-1.53 \pm 4.610$ & & \\
\hline Refreshing & No & 207 & $-0.10 \pm 4.377$ & 0.524 & 0.601 & 137 & $-1.53 \pm 4.551$ & - & - \\
\hline & Yes & 4 & $-1.25 \pm 2.500$ & & & 0 & - & & \\
\hline Relieving sorrow and boredom & No & 165 & $-0.42 \pm 4.440$ & -1.948 & 0.038 & 132 & $-1.36 \pm 3.554$ & 2.376 & 0.019 \\
\hline & Yes & 46 & $0.98 \pm 3.850$ & & & 5 & $-6.20 \pm 16.193$ & & \\
\hline Entertaining & No & 159 & $-0.03 \pm 4.355$ & 0.506 & 0.613 & 133 & $-1.56 \pm 4.565$ & -0.348 & 0.728 \\
\hline & Yes & 52 & $-0.38 \pm 4.362$ & & & 4 & $-0.75 \pm 4.573$ & & \\
\hline Social and business activities & No & 194 & $-0.27 \pm 4.315$ & -1.755 & 0.081 & 136 & $-1.54 \pm 4.566$ & -0.337 & 0.737 \\
\hline & Yes & 17 & $1.65 \pm 4.471$ & & & 1 & 0.00 & & \\
\hline Imitating out of curiosity & No & 209 & $-0.12 \pm 4.370$ & -0.039 & 0.969 & 137 & $-1.53 \pm 4.551$ & - & - \\
\hline & Yes & 2 & $0.00 \pm 0.000$ & & & 0 & - & & \\
\hline Reliving pressure & No & 196 & $-0.14 \pm 4.429$ & -0.233 & 0.816 & 136 & $-1.29 \pm 3.536$ & 9.499 & $<$ \\
\hline & Yes & 15 & $0.13 \pm 3.226$ & & & 1 & -35.00 & & \\
\hline Other reasons (addiction to smoking, & No & 139 & $-0.63 \pm 4.612$ & -2.397 & 0.017 & 134 & $-1.57 \pm 4.596$ & -3.947 & $<$ \\
\hline & Yes & 72 & $0.87 \pm 3.619$ & & & 3 & $0.00 \pm 0.000$ & & \\
\hline Degree of nicotine dependence before & Light & 82 & $-0.17 \pm 3.917$ & -0.255 & 0.799 & 91 & $-1.31 \pm 4.437$ & 2.451 & 0.016 \\
\hline & Moderate & 101 & $-0.12 \pm 4.771$ & & & 37 & $-1.14 \pm 3.699$ & & \\
\hline & & & Page 9/19 & & & & & & \\
\hline
\end{tabular}




\begin{tabular}{|c|c|c|c|c|c|c|c|c|c|}
\hline & Heavy & 28 & $0.05 \pm 4.088$ & & & 9 & $-5.35 \pm 7.168$ & & \\
\hline \multirow{3}{*}{$\begin{array}{l}\text { Degree of nicotine dependence during } \\
\text { the epidemic }\end{array}$} & Light & 159 & $-0.18 \pm 4.422$ & \multirow[t]{3}{*}{-0.071} & \multirow[t]{3}{*}{0.943} & 130 & $-1.26 \pm 4.116$ & \multirow[t]{3}{*}{-} & \multirow[t]{3}{*}{-} \\
\hline & Moderate & 49 & $0.08 \pm 4.281$ & & & 7 & $-6.57 \pm 8.600$ & & \\
\hline & Heavy & 3 & $0.00 \pm 0.000$ & & & 0 & - & & \\
\hline \multirow{5}{*}{$\begin{array}{l}\text { Assessment of willingness to quit } \\
\text { smoking before the epidemic }\end{array}$} & Very low & 29 & $0.45 \pm 5.110$ & \multirow[t]{5}{*}{0.173} & \multirow[t]{5}{*}{0.864} & 21 & $-0.65 \pm 2.065$ & \multirow[t]{5}{*}{3.950} & \multirow[t]{5}{*}{0.001} \\
\hline & $\begin{array}{l}\text { Relatively } \\
\text { low }\end{array}$ & 67 & $-0.36 \pm 4.727$ & & & 70 & $-1.51 \pm 4.991$ & & \\
\hline & Moderate & 85 & $-0.47 \pm 3.869$ & & & 34 & $-2.09 \pm 5.018$ & & \\
\hline & $\begin{array}{l}\text { Relatively } \\
\text { strong }\end{array}$ & 26 & $1.00 \pm 4.252$ & & & 11 & $-0.93 \pm 3.072$ & & \\
\hline & Very strong & 4 & $0.00 \pm 0.000$ & & & 1 & -9.00 & & \\
\hline \multirow{5}{*}{$\begin{array}{l}\text { Assessment of willingness to quit } \\
\text { smoking during the epidemic }\end{array}$} & Very low & 12 & $-2.38 \pm 3.875$ & \multirow[t]{5}{*}{-1.529} & \multirow[t]{5}{*}{0.129} & 3 & $-6.67 \pm 6.506$ & \multirow[t]{5}{*}{-3.198} & \multirow[t]{5}{*}{0.002} \\
\hline & $\begin{array}{l}\text { Relatively } \\
\text { low }\end{array}$ & 13 & $-0.54 \pm 5.206$ & & & 4 & $-0.75 \pm 1.500$ & & \\
\hline & Moderate & 22 & $1.82 \pm 4.532$ & & & 24 & $-2.36 \pm 5.476$ & & \\
\hline & $\begin{array}{l}\text { Relatively } \\
\text { strong }\end{array}$ & 54 & $0.23 \pm 3.970$ & & & 37 & $-1.63 \pm 6.177$ & & \\
\hline & Very strong & 110 & $-0.38 \pm 4.341$ & & & 69 & $-1.012 \pm 2.830$ & & \\
\hline
\end{tabular}

\section{Analysis of factors related to the idea of relapsing of the two groups}

For people who have not quit smoking, the idea of relapsing is related to the psychological state of calmness and the need to relieve sorrow and boredom through smoking, the distribution difference is statistically significant (all $\mathrm{P}<0.05$ ), see Table 7. Among people who reported having quit smoking, the idea of relapsing is associated with such factors as depression, relieving sorrow and boredom, and reliving pressure, the distribution difference is of statistical significance (all $\mathrm{P}<0.05$ ). 
Table 7

Factors Related to the Idea of Relapsing of Subjects during the epidemic of COVID-19 [n(\%)]

\begin{tabular}{|c|c|c|c|c|c|c|c|c|c|}
\hline \multirow[t]{2}{*}{ Questions } & \multirow[t]{2}{*}{ Degree } & \multicolumn{4}{|c|}{ Persistent smokers $(\mathrm{n}=211)$} & \multicolumn{4}{|c|}{ Ex-smokers $(n=137)$} \\
\hline & & No & Yes & $z / x^{2}$ & $p$ & No & Yes & $Z / \chi^{2}$ & $P$ \\
\hline \multirow{4}{*}{$\begin{array}{l}\text { The length of staying at home } \\
\text { per day in the past two weeks }\end{array}$} & Almost all day & $117(63.2)$ & 18(69.2) & \multirow[t]{4}{*}{0.390} & \multirow[t]{4}{*}{0.942} & $76(62.8)$ & $11(68.8)$ & \multirow[t]{4}{*}{0.750} & \multirow[t]{4}{*}{0.861} \\
\hline & $\begin{array}{l}80 \%-90 \% \text { of } \\
\text { the day }\end{array}$ & $16(8.6)$ & $2(7.7)$ & & & 16(13.2) & $1(6.3)$ & & \\
\hline & $\begin{array}{l}50 \%-80 \% \text { of } \\
\text { the day }\end{array}$ & 24(13.0) & $3(11.5)$ & & & 14(11.6) & $2(12.5)$ & & \\
\hline & $\begin{array}{l}\text { Less than } \\
50 \% \text { of the } \\
\text { day }\end{array}$ & 28(15.1) & $3(11.5)$ & & & 15(12.4) & $2(12.5)$ & & \\
\hline \multirow[t]{2}{*}{ Suspected } & No & 182(98.4) & $25(96.2)$ & \multirow[t]{2}{*}{0.489} & \multirow[t]{2}{*}{0.484} & 121(100.0) & 16(100.0) & \multirow[t]{2}{*}{-} & \multirow[t]{2}{*}{-} \\
\hline & Yes & $3(1.6)$ & $1(3.8)$ & & & $0(0.0)$ & $0(0.0)$ & & \\
\hline \multirow[t]{2}{*}{ Worried } & No & 147(79.5) & 19(73.1) & \multirow[t]{2}{*}{0.553} & \multirow[t]{2}{*}{0.457} & 109(90.1) & 13(81.3) & \multirow[t]{2}{*}{0.406} & \multirow[t]{2}{*}{0.524} \\
\hline & Yes & $38(20.5)$ & $7(26.9)$ & & & 12(9.9) & $3(18.8)$ & & \\
\hline \multirow[t]{2}{*}{ Panic } & No & 177(95.7) & 24(92.3) & \multirow[t]{2}{*}{0.070} & \multirow[t]{2}{*}{0.792} & $115(95.0)$ & 16(100.0) & \multirow[t]{2}{*}{1.526} & \multirow[t]{2}{*}{0.217} \\
\hline & Yes & $8(4.3)$ & $2(7.7)$ & & & $6(5.0)$ & $0(0.0)$ & & \\
\hline \multirow[t]{2}{*}{ Depressed } & No & 172(93.0) & 21(80.8) & \multirow[t]{2}{*}{2.928} & 0.087 & 116(95.9) & 13(81.3) & 3.876 & 0.049 \\
\hline & Yes & $13(7.0)$ & $5(19.2)$ & & & $5(4.1)$ & $3(18.8)$ & & \\
\hline Calm & No & $99(53.5)$ & $24(92.3)$ & 14.111 & $<$ & $53(43.8)$ & $7(43.8)$ & 0.000 & 0.997 \\
\hline & Yes & $86(46.5)$ & $2(7.7)$ & & & $68(56.2)$ & $9(56.3)$ & & \\
\hline Almost as usual & No & $38(20.5)$ & $9(34.6)$ & 2.608 & 0.106 & $14(11.6)$ & $4(25.0)$ & 1.212 & 0.271 \\
\hline & Yes & 147(79.5) & $17(65.4)$ & & & 107(88.4) & $12(75.0)$ & & \\
\hline Refreshing & No & 181(97.8) & $26(100.0)$ & 1.063 & 0.303 & $121(100.0)$ & $16(100.0)$ & - & - \\
\hline & Yes & $4(2.2)$ & $0(0.0)$ & & & $0(0.0)$ & $0(0.0)$ & & \\
\hline Relieving sorrow and boredom & No & 151(81.6) & $14(53.8)$ & 10.316 & 0.001 & $120(99.2)$ & $12(75.0)$ & 13.343 & $<$ \\
\hline & Yes & $34(18.4)$ & $12(46.2)$ & & & $1(0.8)$ & $4(25.0)$ & & \\
\hline Entertaining & No & $141(76.2)$ & 18(69.2) & 0.599 & 0.439 & 119(98.3) & 14(87.5) & 5.866 & 0.015 \\
\hline & Yes & $44(23.8)$ & $8(30.8)$ & & & 2(1.7) & $2(12.5)$ & & \\
\hline Social and business activities & No & 170(91.9) & $24(92.3)$ & 0.005 & 0.942 & 120(99.2) & $16(100.0)$ & 0.249 & 0.618 \\
\hline & Yes & $15(8.1)$ & $2(7.7)$ & & & $1(0.8)$ & $0(0.0)$ & & \\
\hline Imitating out of curiosity & No & 184(99.5) & 25(96.2) & 1.703 & 0.192 & 121(100.0) & $16(100.0)$ & - & - \\
\hline & Yes & $1(0.5)$ & $1(3.8)$ & & & $0(0.0)$ & $0(0.0)$ & & \\
\hline Relieving pressure & No & 173(93.5) & 23(88.5) & 0.282 & 0.595 & 121(100.0) & 15(93.8) & 4.351 & 0.037 \\
\hline & Yes & 12(6.5) & $3(11.5)$ & & & $0(0.0)$ & $1(6.3)$ & & \\
\hline Other reasons (addiction to & No & $121(65.4)$ & 18(69.2) & 0.148 & 0.700 & 119(98.3) & 15(93.8) & 1.003 & 0.316 \\
\hline & Yes & $64(34.6)$ & $8(30.8)$ & & & $2(1.7)$ & $1(6.3)$ & & \\
\hline Degree of nicotine dependence & Light & $70(37.8)$ & $12(46.2)$ & -0.277 & 0.782 & $81(66.9)$ & $10(62.5)$ & -0.315 & 0.752 \\
\hline & Moderate & $92(49.7)$ & $9(34.6)$ & & & $32(26.4)$ & $5(31.3)$ & & \\
\hline & Heavy & 23(12.4) & $5(19.2)$ & & & $8(6.6)$ & $1(6.3)$ & & \\
\hline Degree of nicotine dependence & Light & $140(75.7)$ & 19(73.1) & -0.358 & 0.721 & 116(95.9) & 14(87.5) & -1.423 & 0.155 \\
\hline & Moderate & $43(23.2)$ & $6(23.1)$ & & & $5(4.1)$ & $2(12.5)$ & & \\
\hline
\end{tabular}




\begin{tabular}{|c|c|c|c|c|c|c|c|c|c|}
\hline & Heavy & $2(1.1)$ & $1(3.8)$ & & & $0(0.0)$ & $0(0.0)$ & & \\
\hline \multirow{5}{*}{$\begin{array}{l}\text { Assessment of willingness to } \\
\text { quit smoking before the } \\
\text { epidemic }\end{array}$} & Very low & $27(14.6)$ & $2(7.7)$ & \multirow[t]{5}{*}{-1.064} & \multirow[t]{5}{*}{0.287} & 19(15.7) & $2(12.5)$ & \multirow[t]{5}{*}{-1.445} & \multirow[t]{5}{*}{0.148} \\
\hline & Relatively low & $57(30.8)$ & $10(38.5)$ & & & $64(52.9)$ & $6(37.5)$ & & \\
\hline & Moderate & $79(42.7)$ & $6(23.1)$ & & & $29(24.0)$ & $5(31.3)$ & & \\
\hline & $\begin{array}{l}\text { Relatively } \\
\text { strong }\end{array}$ & 18(9.7) & $8(30.8)$ & & & $9(7.4)$ & $2(12.5)$ & & \\
\hline & Very strong & $4(2.2)$ & $0(0.0)$ & & & $0(0.0)$ & $1(6.3)$ & & \\
\hline \multirow{5}{*}{$\begin{array}{l}\text { Assessment of willingness to } \\
\text { quit smoking during the } \\
\text { epidemic }\end{array}$} & Very low & $10(5.4)$ & $2(7.7)$ & \multirow[t]{5}{*}{-0.438} & \multirow[t]{5}{*}{0.661} & $3(2.5)$ & $0(0.0)$ & \multirow[t]{5}{*}{-1.201} & \multirow[t]{5}{*}{0.230} \\
\hline & Relatively low & $13(7.0)$ & $0(0.0)$ & & & $4(3.3)$ & $0(0.0)$ & & \\
\hline & Moderate & $20(10.8)$ & $2(7.7)$ & & & $22(18.2)$ & $2(12.5)$ & & \\
\hline & $\begin{array}{l}\text { Relatively } \\
\text { strong }\end{array}$ & $46(24.9)$ & $8(30.8)$ & & & $33(27.3)$ & $4(25.0)$ & & \\
\hline & Very strong & $96(51.9)$ & $14(53.8)$ & & & $59(48.8)$ & $10(62.5)$ & & \\
\hline
\end{tabular}

\section{Analysis of factors related to nicotine dependence of the two groups}

Among people who have not quit smoking, the degree of nicotine dependence during the epidemic is related to the psychological state of depression, the degree of nicotine dependence before the epidemic, and the willingness to quit smoking before and during the epidemic, and the distribution difference is statistically significant (all $\mathrm{P}<0.05$ ), See Table 8. Among people who reported having quit smoking, the degree of nicotine dependence during the epidemic is related to the daily length of staying at home, the psychological state of calmness, addiction and smoking habits, and the degree of nicotine dependence before the epidemic, and the distribution difference is statistically significant (all $\mathrm{P}<0.05$ ).

Table 8

Factors Related to the Degree of Nicotine Dependence of Subjects during the epidemic of COVID-19 [n(\%)] 


\begin{tabular}{|c|c|c|c|c|c|c|c|c|c|c|c|}
\hline \multirow[t]{2}{*}{ Questions } & \multirow[t]{2}{*}{ Degree } & \multicolumn{5}{|c|}{ Persistent smokers $(n=211)$} & \multicolumn{5}{|c|}{ Ex-smokers $(n=137)$} \\
\hline & & Light & Moderate & Heavy & $Z / \chi^{2}$ & $p$ & Light & Moderate & Heavy & $Z / \boldsymbol{x}^{2}$ & $p$ \\
\hline \multirow{4}{*}{$\begin{array}{l}\text { The length } \\
\text { of staying } \\
\text { at home per } \\
\text { day in the } \\
\text { past two } \\
\text { weeks }\end{array}$} & $\begin{array}{l}\text { Almost } \\
\text { all day }\end{array}$ & $103(64.8)$ & $30(61.2)$ & $2(66.7)$ & \multirow[t]{4}{*}{0.582} & \multirow[t]{4}{*}{0.748} & $80(61.5)$ & $7(100.0)$ & $0(0.0)$ & \multirow[t]{4}{*}{3.962} & \multirow[t]{4}{*}{0.047} \\
\hline & $\begin{array}{l}80 \%-90 \% \\
\text { of the } \\
\text { day }\end{array}$ & $15(9.4)$ & $3(6.1)$ & $0(0.0)$ & & & 17(13.1) & $0(0.0)$ & $0(0.0)$ & & \\
\hline & $\begin{array}{l}50 \%-80 \% \\
\text { of the } \\
\text { day }\end{array}$ & $20(12.6)$ & $6(12.2)$ & 1(33.3) & & & $16(12.3)$ & $0(0.0)$ & $0(0.0)$ & & \\
\hline & $\begin{array}{l}\text { Less } \\
\text { than } 50 \% \\
\text { of the } \\
\text { day }\end{array}$ & $21(13.2)$ & $10(20.4)$ & $0(0.0)$ & & & 17(13.1) & $0(0.0)$ & $0(0.0)$ & & \\
\hline \multirow{2}{*}{$\begin{array}{l}\text { Although } \\
\text { you have } \\
\text { quit } \\
\text { smoking, do } \\
\text { you want to } \\
\text { smoke } \\
\text { because of } \\
\text { the } \\
\text { epidemic } \\
\text { (namely } \\
\text { relapse) }\end{array}$} & No & 140(88.1) & $43(87.8)$ & $2(66.7)$ & \multirow[t]{2}{*}{1.241} & \multirow[t]{2}{*}{0.538} & 116(89.2) & $5(71.4)$ & $0(0.0)$ & \multirow[t]{2}{*}{2.026} & \multirow[t]{2}{*}{0.155} \\
\hline & Yes & 19(11.9) & $6(12.2)$ & 1(33.3) & & & 14(10.8) & $2(28.6)$ & $0(0.0)$ & & \\
\hline \multirow[t]{2}{*}{ Suspected } & No & 155(97.5) & 49(100.0) & $3(100.0)$ & \multirow[t]{2}{*}{1.327} & \multirow[t]{2}{*}{0.515} & $130(100.0)$ & $7(100.0)$ & $0(0.0)$ & \multirow[t]{2}{*}{0.000} & \multirow[t]{2}{*}{1.000} \\
\hline & Yes & $4(2.5)$ & $0(0.0)$ & $0(0.0)$ & & & $0(0.0)$ & $0(0.0)$ & $0(0.0)$ & & \\
\hline \multirow[t]{2}{*}{ Worried } & No & 125(78.6) & $40(81.6)$ & 1(33.3) & \multirow[t]{2}{*}{3.913} & \multirow[t]{2}{*}{0.141} & 117(90.0) & $5(71.4)$ & $0(0.0)$ & \multirow[t]{2}{*}{2.332} & \multirow[t]{2}{*}{0.127} \\
\hline & Yes & $34(21.4)$ & $9(18.4)$ & $2(66.7)$ & & & $13(10.0)$ & $2(28.6)$ & $0(0.0)$ & & \\
\hline \multirow[t]{2}{*}{ Panic } & No & 152(95.6) & $46(93.9)$ & $3(100.0)$ & \multirow[t]{2}{*}{0.395} & \multirow[t]{2}{*}{0.821} & 124(95.4) & $7(100.0)$ & $0(0.0)$ & 0.335 & 0.562 \\
\hline & Yes & $7(4.4)$ & $3(6.1)$ & $0(0.0)$ & & & $6(4.6))$ & $0(0.0)$ & $0(0.0)$ & & \\
\hline Depressed & No & 141(88.7) & 49(100.0) & $3(100.0)$ & 6.405 & 0.041 & 123(94.6) & $6(85.7)$ & $0(0.0)$ & 0.950 & 0.330 \\
\hline & Yes & 18(11.3) & $0(0.0)$ & $0(0.0)$ & & & $7(5.4)$ & $1(14.3)$ & $0(0.0)$ & & \\
\hline Calm & No & $96(60.4)$ & $26(53.1)$ & 1(33.3) & 1.597 & 0.450 & $54(41.5)$ & $6(85.7)$ & $0(0.0)$ & 5.228 & 0.022 \\
\hline & Yes & $63(39.6)$ & $23(46.9)$ & $2(66.7)$ & & & $76(58.5)$ & $1(14.3)$ & $0(0.0)$ & & \\
\hline Almost as & No & $37(23.3)$ & $9(18.4)$ & 1(33.3) & 0.732 & 0.694 & $16(12.3)$ & $2(28.6)$ & $0(0.0)$ & 1.528 & 0.216 \\
\hline & Yes & 12(76.7) & $40(81.6)$ & $2(66.7)$ & & & 114(87.7) & $5(71.4)$ & $0(0.0)$ & & \\
\hline Refreshing & No & 156(98.1) & $48(98.0)$ & $3(100.0)$ & 0.063 & 0.969 & 130(100.0) & 7(100.0) & $0(0.0)$ & 0.000 & 1.000 \\
\hline & Yes & $3(1.9)$ & $1(2.0)$ & $0(0.0)$ & & & $0(0.0)$ & $0(0.0)$ & $0(0.0)$ & & \\
\hline Relieving & No & 122(76.7) & 41(83.7) & $2(66.7)$ & 1.291 & 0.524 & 126(96.9) & $6(85.7)$ & $0(0.0)$ & 2.356 & 0.125 \\
\hline boredom & Yes & $37(23.3)$ & $8(16.3)$ & 1(33.3) & & & $4(3.1)$ & $1(14.3)$ & $0(0.0)$ & & \\
\hline Entertaining & No & 117(73.6) & $40(81.6)$ & $2(66.7)$ & 1.423 & 0.491 & 126(96.9) & $7(100.0)$ & $0(0.0)$ & 0.220 & 0.639 \\
\hline & Yes & $42(26.4)$ & $9(18.4)$ & 1(33.3) & & & $4(3.1)$ & $0(0.0)$ & $0(0.0)$ & & \\
\hline Social and & No & 144(90.6) & $47(95.9)$ & $3(100.0)$ & 1.707 & 0.426 & 129(99.2) & $7(100.0)$ & $0(0.0)$ & 0.054 & 0.817 \\
\hline activities & Yes & $15(9.4)$ & $2(4.1)$ & $0(0.0)$ & & & $1(0.8)$ & $0(0.0)$ & $0(0.0)$ & & \\
\hline Imitating & No & 158(99.4) & 48(98.0) & $3(100.0)$ & 0.820 & 0.663 & $130(100.0)$ & $7(100.0)$ & $0(0.0)$ & 0.000 & 1.000 \\
\hline curiosity & Yes & $1(0.6)$ & $1(2.0)$ & $0(0.0)$ & & & $0(0.0)$ & $0(0.0)$ & $0(0.0)$ & & \\
\hline Relieving & No & 147(92.5) & 46(93.9) & $3(100.0)$ & 0.346 & 0.841 & 129(99.2) & 7(100.0) & $0(0.0)$ & 0.054 & 0.817 \\
\hline & Yes & $12(7.5)$ & $3(6.1)$ & $0(0.0)$ & & & $1(0.8)$ & $0(0.0)$ & $0(0.0)$ & & \\
\hline
\end{tabular}




\begin{tabular}{|c|c|c|c|c|c|c|c|c|c|c|c|}
\hline \multirow{2}{*}{$\begin{array}{l}\text { Other } \\
\text { reasons } \\
\text { (addiction } \\
\text { to smoking, } \\
\text { habits, etc.) }\end{array}$} & No & 108(67.9) & $28(57.1)$ & $3(100.0)$ & \multirow[t]{2}{*}{3.497} & \multirow[t]{2}{*}{0.174} & $129(99.2)$ & $5(71.4)$ & $0(0.0)$ & \multirow[t]{2}{*}{23.796} & \multirow[t]{2}{*}{$<0.001$} \\
\hline & Yes & $51(32.1)$ & $21(42.9)$ & $0(0.0)$ & & & $1(0.8)$ & $2(28.6)$ & $0(0.0)$ & & \\
\hline \multirow{3}{*}{$\begin{array}{l}\text { Degree of } \\
\text { nicotine } \\
\text { dependence } \\
\text { before the } \\
\text { epidemic }\end{array}$} & Light & $74(46.5)$ & $8(16.3)$ & $0(0.0)$ & \multirow[t]{3}{*}{19.094} & \multirow[t]{3}{*}{$<0.001$} & $90(69.2)$ & $1(14.3)$ & $0(0.0)$ & \multirow[t]{3}{*}{10.065} & \multirow[t]{3}{*}{0.002} \\
\hline & Moderate & $69(43.4)$ & $31(63.3)$ & 1(33.3) & & & $33(25.4)$ & $4(57.1)$ & $0(0.0)$ & & \\
\hline & Heavy & 16(10.1) & $10(20.4)$ & $2(66.7)$ & & & $7(5.4)$ & 2(28.6) & $0(0.0)$ & & \\
\hline \multirow{5}{*}{$\begin{array}{l}\text { Assessment } \\
\text { of } \\
\text { willingness } \\
\text { to quit } \\
\text { smoking } \\
\text { before the } \\
\text { epidemic }\end{array}$} & Very low & $25(15.7)$ & $4(8.2)$ & $0(0.0)$ & \multirow[t]{5}{*}{15.045} & \multirow[t]{5}{*}{0.001} & $19(14.6)$ & $2(28.6))$ & $0(0.0)$ & \multirow[t]{5}{*}{0.000} & \multirow[t]{5}{*}{1.000} \\
\hline & $\begin{array}{l}\text { Relatedly } \\
\text { low }\end{array}$ & $54(34.0)$ & $13(26.5)$ & $0(0.0)$ & & & 68(52.3) & $2(28.6)$ & $0(0.0)$ & & \\
\hline & Moderate & $65(40.9)$ & $20(40.8)$ & $0(0.0)$ & & & $32(24.6)$ & 2(28.6) & $0(0.0)$ & & \\
\hline & $\begin{array}{l}\text { Relatively } \\
\text { strong }\end{array}$ & 14(8.8) & $11(22.4)$ & 1(33.3) & & & 10(7.7)) & $1(14.3)$ & $0(0.0)$ & & \\
\hline & $\begin{array}{l}\text { Very } \\
\text { strong }\end{array}$ & $1(0.6)$ & $1(2.0)$ & 2(66.7) & & & $1(0.8)$ & $0(0.0)$ & $0(0.0)$ & & \\
\hline \multirow{5}{*}{$\begin{array}{l}\text { Assessment } \\
\text { of } \\
\text { willingness } \\
\text { to quit } \\
\text { smoking } \\
\text { during the } \\
\text { epidemic }\end{array}$} & Very low & $12(7.5)$ & $0(0.0)$ & $0(0.0)$ & \multirow[t]{5}{*}{0.962} & \multirow[t]{5}{*}{0.618} & $3(2.3)$ & $0(0.0)$ & $0(0.0)$ & \multirow[t]{5}{*}{0.018} & \multirow[t]{5}{*}{0.894} \\
\hline & $\begin{array}{l}\text { Relatedly } \\
\text { low }\end{array}$ & $10(6.3)$ & $3(6.1)$ & $0(0.0)$ & & & $4(3.1)$ & $0(0.0)$ & $0(0.0)$ & & \\
\hline & Moderate & 13(8.2) & $9(18.4)$ & $0(0.0)$ & & & 21(16.2) & $3(42.9)$ & $0(0.0)$ & & \\
\hline & $\begin{array}{l}\text { Relatively } \\
\text { strong }\end{array}$ & $36(22.6)$ & 16(32.7) & $2(66.7)$ & & & $37(28.5)$ & $0(0.0)$ & $0(0.0)$ & & \\
\hline & $\begin{array}{l}\text { Very } \\
\text { strong }\end{array}$ & $88(55.3)$ & 21(42.9) & 1(33.3) & & & $65(50.0)$ & $4(57.1)$ & $0(0.0)$ & & \\
\hline
\end{tabular}

\section{Analysis of factors related to the willingness to quit smoking during the epidemic of the two groups}

Among people who have not quit smoking, the willingness to quit smoking during the epidemic is related to the length of staying at home per day and the degree of nicotine dependence during the epidemic, and the distribution difference is statistically significant (all $\mathrm{P}<0.05$ ), see Table 9 . Among people who reported having quit smoking, the willingness to quit during the epidemic is related to entertaining and addiction and smoking habits, and their distribution difference is statistically significant (all $\mathrm{P}<0.05$ ). 


\begin{tabular}{|c|c|c|c|c|c|c|c|c|c|c|c|c|c|c|c|}
\hline \multirow[b]{2}{*}{ Questions } & \multirow[b]{2}{*}{ Derree } & \multicolumn{7}{|c|}{ Persistant momorers (1-211) } & \multicolumn{7}{|c|}{ Ex $x$ makeres $(n-137)$} \\
\hline & & Vayjlow & $\begin{array}{c}\text { Reatruey } \\
\text { low }\end{array}$ & Modatats & $\begin{array}{c}\text { Relatively } \\
\text { stong }\end{array}$ & $\begin{array}{l}\text { Very } \\
\text { stong } \\
\end{array}$ & $z z^{2}$ & $p$ & $\begin{array}{l}\text { Vers } \\
\text { Low }\end{array}$ & $\begin{array}{c}\text { Relatively } \\
\text { low }\end{array}$ & Moderate & $\begin{array}{c}\text { Relativiey } \\
\text { strong }\end{array}$ & $\begin{array}{l}\text { Yeny } \\
\text { stroug } \\
\end{array}$ & $z x^{2}$ & ${ }^{p}$ \\
\hline & Alluost al day & $3(25.0)$ & $10(76.9)$ & $12(5+5)$ & $32[59.3]$ & $78(70.9)$ & \multirow{4}{*}{ 11.747 } & & $2(6.7)$ & $2(560.0)$ & $17(70.8)$ & $24(649)$ & 42(60.9) & & \\
\hline The length of fasing z th home & $80 \%-90 \%$ of the day & $325.0)$ & $2(15.4)$ & 29.1) & $8(48)$ & 32.7) & & (1) & $0(0.0)$ & $(00)$ & $4(167)$ & $4(10.8)$ & $9(130)$ & & \\
\hline per das in the rast two wets & $50 \%-80 \%$ of the day & 2:16.7) & $(00)$ & 29.1) & 6(1.1.1) & $17(15.5)$ & & 0.019 & $1(3.3 .3)$ & $1(25.0)$ & $2(83)$ & $4(10.8)$ & $8(11.6)$ & 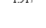 & . \\
\hline & Les then $5 \%$ of the dy & 4(31.3) & $1(7.7)$ & 6.27.3) & $8(14.8)$ & 12,1099) & & & $0(0.0)$ & 125:0) & ${ }^{1(42)}$ & $5(13.5)$ & $10(14.5)$ & & \\
\hline Although you hare auit & No & $10(839)$ & $13(100.0)$ & 2090999 & $46(1832)$ & 96(673) & & & $3(100.0)$ & $4(100.0)$ & $2201.7)$ & 3388927 & $59855]$ & & \\
\hline $\begin{array}{l}\text { smoling, doyou wart to } \\
\text { smoke because of the } \\
\text { evidemic (namely relpesse) }\end{array}$ & Yes & $2(16.7)$ & $0(0,0)$ & 29.1) & $8(148)$ & ${ }^{14(1227)}$ & 2564 & 0.633 & $0(0.0)$ & $(0,0)$ & 2(83) & $4(10.8)$ & 10(1.5.5) & 1.730 & 0.785 \\
\hline SWunted & No & 122(100.0.0) & $13(100.9)$ & $22(100.0)$ & $51(94.4]$ & 109(9.1.1) & & & $3(100.0)$ & $4(100.0)$ & $24(100.0)$ & $37(100.0)$ & $69(100.0)$ & & 1000 \\
\hline susperced & Yes & $0(0,0)$ & $0(00)$ & $\alpha(0.0)$ & $3(5.9)$ & $1(0.9)$ & 5.348 & 0.253 & $0(0.0)$ & $0(0.0)$ & $(0.0)$ & $\alpha(0.0)$ & $00.0 .9)$ & 000 & - \\
\hline Woried & No & 12(1000.0) & 10(76.9) & $16(72.7)$ & $43(79.6)$ & 85(773) & 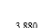 & 0403 & $x^{2(6.7)}$ & $4(100.0)$ & 23(95.8) & 31(89.8) & 6228999) & & \\
\hline we & Yes & $(0,0)$ & 3(23.1) & 627.73) & $11(20.4)$ & $25(227)$ & $=0.00$ & $0 .+23$ & $1(3.3 .3)$ & $\theta(0.0)$ & 1(42) & $6(162)$ & $7(0.1)$ & +.233 & 0.315 \\
\hline Pawic & No & 12(100.0.) & $13(100.0)$ & 2090.99 & $51(94,4)$ & $105(9.5 .5)$ & 2.245 & 0.691 & $3(100.0)$ & $4(100.0)$ & $23(9.8 .8)$ & $3699.3)$ & $65594.2)$ & 0.986 & 0.925 \\
\hline & Yes & $0(0.0)$ & $0(0.0)$ & $29.1)$ & 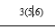 & $5(4)$. & & & $0(0.0)$ & $g(0.0)$ & $1(42)$ & 12.7. & $4(5, s)$ & & \\
\hline & No & 12(100.0.) & ${ }^{12(29.23)}$ & ${ }^{18(81.8)}$ & $4990.7)$ & 102(29.7) & & & $2(6.7)$ & $4(100.0)$ & $23(9.8)$ & $33(94.6)$ & $65[94.2)$ & 4475 & \\
\hline Depressed & Yes & $x(0)$ & $1(77)$ & 4(182) & $5(93))$ & $8(7.3)$ & 3.998 & ${ }^{0.406}$ & $1(3.3 .3)$ & $(0,0)$ & $1(42)$ & $2(5,4)$ & $4(5.8)$ & 4.475 & 0.346 \\
\hline Cam & No & 4(3.3.3) & $4(30.8)$ & $16(72.7)$ & 32[59.3] & $67(60.9)$ & 0.907 & 0054 & $1(33.3)$ & $2(50.0)$ & $10(4.17)$ & $15(40.5)$ & $32(46.4)$ & 0.589 & 0.65 \\
\hline & Yes & $866.7)$ & 9(69:2) & $6027.3)$ & 2240.77 & 43,39.1) & $=2.25$ & 0.004 & $z(6.7)$ & $2(50.0)$ & 14(58.5) & 22:59.5) & $37(5.6)$ & 0.032 & 5.903 \\
\hline Alwest the & No & $a(00)$ & $4(30.8)$ & $8(364)$ & 11(20.4) & 24(21.18) & :598 $>2>$ & 01989 & $1(3.3)$ & $a(a)$ & $2(83)$ & $5(135)$ & $10(145)$ & & \\
\hline Allmost 13 sual & Yes & 12(100.0.) & $9(69.2)$ & 14(6.3.6) & 43(79.6.6) & 86(7822) & 6.598 & 0.159 & $2(66.7)$ & $4(100.0)$ & $22(91.7)$ & $32 \times 8.5)$ & $59(8.5)$ & 2.251 & 0.638 \\
\hline Befalving & No & 11(91.) & $13(100.09)$ & $22(100.9)$ & $5339.1 .1]$ & $108(98.2)$ & 3.399 & 0503 & $3(100.0)$ & $4(100.0)$ & $24(100.0 .9)$ & $37(000.0)$ & $69(100.0)$ & 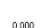 & 1000 \\
\hline Nerentug & Yoas & 1(83) & $(00)$ & $(0,0)$ & $1(1.9)$ & 2(1.8) & (j.359 & 0.003 & $0(0.0)$ & o(cao) & $(\infty, 0)$ & $\alpha(0.0)$ & $0(0.0)$ & 0.000 & 1.000 \\
\hline Relieirigs sorow and & No & 11(91.) & $9(6.2)$ & $18(8.1 .8 ;$ & 42(77.8) & 85(77.3) & 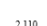 & 0.715 & $2(6.7)$ & $4(100.0)$ & $24(100.0)$ & $35(94.6)$ & $67797.11)$ & 8077 & 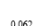 \\
\hline bereleom & Yes & $1(83)$ & $4(30.8)$ & $4(182)$ & 12(22.2) & 25(2227) & 1.10 & 0,10 & $1(3.3 .3)$ & $0(0,0)$ & $(0.0)$ & $25.54)$ & 2(2.9) & . & . \\
\hline & No & $8(66.7)$ & $7(5.8)$ & $18(8.1 .8)$ & 40(77.1) & 86(7822) & & & $1^{1(3.3 .3)}$ & $4(100.0)$ & $24(100.0)$ & $36(99.3)$ & 68998.6) & & \\
\hline Entatrtimng & Yes & 4.33.3) & 6446.2) & $4(18.2)$ & 1425.99 & 24(21.8) & 4.720 & 0.317 & $2(6,0)$ & $o(0.0)$ & $\alpha(0.0)$ & $12.7)$ & 1(1.4) & 4..661 & 80.001 \\
\hline Social Int laverines & No & 12z(100.0.0) & $13(100.9)$ & $21(9.5)$ & $4990.7]$ & $9990.0)$ & 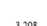 & 0.50 & $3(100.0)$ & $4(100.0)$ & $23(95.8)$ & $37(100.0)$ & $69(100.0)$ & (2) & م \\
\hline 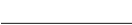 & Yes & $0(0,0)$ & $0(0.0)$ & ${ }^{1(4.5)}$ & $5(0.3)$ & $11(10.0)$ & 2.200 & 0.54 & $0(0.0)$ & $0(0.0)$ & ${ }_{1(42)}$ & $\alpha 0.0)$ & $0(0.0)$ & 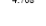 & 3.39 \\
\hline 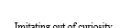 & No & 12000.0) & $13(100.0)$ & $22(100.0)$ & $53(981)$ & $10999.1)$ & & & $3(100.0)$ & $4(100.0)$ & 24(1000.0) & $37(100.0)$ & $69(000.0)$ & & \\
\hline Imithting out of c curiosity & Yes & $0(0.0)$ & $0(0.0)$ & 00.09 & (1..5) & $1(0.9)$ & 0917 & 0.922 & o(co.0) & (0.0) & $010.0)$ & $0(0.0)$ & $00.0)$ & 0.000 & 1.000 \\
\hline Rolt & No & 10(183.3) & 13(100.0) & 20(9099) & $52(963)$ & $10191.8)$ & 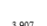 & , & $3(100.0)$ & $4(100.0)$ & $24(100.0)$ & $36(973)$ & $69(100.0)$ & 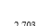 & 0000 \\
\hline . & Yas & $2,(16.7)$ & $0(0.0 .9)$ & 2(9.1) & $2(3.7)$ & $9(8.2)$ & (3) & 年 & $\alpha(0.0)$ & $\alpha 0.0)$ & $0(0.0)$ & 12.7) & $0(0.0)$ & 2003 & 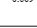 \\
\hline 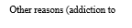 & no & $9(75.0)$ & 1077699) & $10(455)$ & $32(993)$ & ${ }^{78(0.9)}$ & & & $2(66.7)$ & $4(100.0)$ & ${ }^{23(958)}$ & $37(1000)$ & 68986 & & \\
\hline smoking, habits, etc) & Yas & $3(25.0)$ & 3(23.1) & $12(544)$ & $22(40.7)$ & 32(29.1) & ${ }^{7487}$ & 0.112 & $1(33.3)$ & $(0,0.0)$ & 1(4.2) & $0(0.0)$ & 1(1.4) & 15.008 & 0.005 \\
\hline Degese of tiricoine & Light & $3(25.0)$ & $5(8.5)$ & $9(40.9)$ & $15(27.8)$ & $50(4.5 .5)$ & & & $3(100.0)$ & $3(75.0)$ & 19(992) & 24(649) & $+2(60.9)$ & & \\
\hline 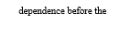 & Moderata & $8(66.7)$ & $5(3.5)$ & $10(455)$ & $30(5.5)$ & $48(43.6)$ & 5246 & 0.263 & $x(0)$ & $1(250)$ & $4(16.7)$ & 13(3.1) & 19(275) & 4.752 & 0.314 \\
\hline epridmic & Hexry & $18.3)$ & 3(23.1) & $3(13.6)$ & $9(16)$. & 12(10.9) & & & $x(0.0)$ & $0(0.0)$ & $1(4.2)$ & $00.0)$ & $8(1.1)$. & & \\
\hline Degree of nivooine & Light & 122000.9) & $107769)$ & 13(59.1) & $36(6, .7)$ & $88(0.0 .0)$ & & & $3(100.9)$ & $4(100.09)$ & $21(87.5)$ & $37(100.0)$ & ${ }^{659942)}$ & & \\
\hline dependerca during the & Modertate & $0(0.0)$ & 3(2.3.1) & $9(40.9)$ & $16(996)$ & $2(19.1)$ & 10.501 & 0.033 & $x(0.0)$ & $\alpha(0,0)$ & $3(125)$ & $0(0.9)$ & 4(5.8) & 5.03 & 0.277 \\
\hline epridmic & Heary & $0(0.0)$ & $0(0.0)$. & $00.0)$ & $2(3.7)$ & $1(0.9)$ & & & $x(0.0)$ & $\alpha 0,0)$ & $00.0 .0)$ & $0(0.0)$ & $000.0)$ & & \\
\hline & Very low & $0(0.0)$ & 177. $)$ & $4(1.82)$ & 7(13.0) & $17(1.5 .5)$ & & & $x(0.0)$ & $\alpha(0.0)$ & ${ }_{1(4.2)}$ & $7(18.9)$ & 13(198) & & \\
\hline 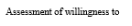 & Roldativaly low & 4(333) & $7(3.3 .8)$ & $5(2.2 .7)$ & ${ }^{14(259)}$ & $37(3.3 .6)$ & & & $1(3.3)$ & $2(50.0)$ & ${ }^{13(342)}$ & ${ }_{19(1.14)}$ & $35(50.7)$ & & \\
\hline quit tmoling befre the & Moderte & $5(4.7)$ & $4(30.8)$ & $10(455)$ & $16(29.6)$ & $50(4.5)$ & 8.859 & 0.065 & $2(6.7)$ & 1250) & $7(29.2)$ & 6(16.2) & $1826.61)$ & 5.121 & 0.275 \\
\hline eppidemic & Relativey ytowng & $3(25.0)$ & 10.7 & 3(13..) & 13(204.1) & $6(5.5)$ & & & o(co.) & 125.0) & $3(12.5)$ & $5(13.5)$ & 22(2.9) & & \\
\hline & Vay trong & $0(0.0)$ & $0(0.0)$ & $0(0.0)$ & 4(C.4) & $0(0.0)$ & & & o(co) & $\alpha(0,0)$ & $0(0.0)$ & $0(0.0)$ & 1(1.4) & & \\
\hline
\end{tabular}

\section{Discussion And Suggestions}

At the beginning of 2020, the epidemic of COVID-19 broke out. In order to prevent the further spread of the epidemic, at the call of the Chinese government and the Beijing municipal government, the public voluntarily stayed at home and isolated themselves based on the principle of "no fairs, no visits, and no gatherings". Previously, people who tried to quit smoking had received 3 to 6 months of intervention service, and some of them had succeeded. The sudden epidemic has changed the motivation, psychological state, and willingness of smokers trying to quit, which has influenced their smoking cessation behavior. This study used the method of propensity score matching to control confounding factors. After matching, we analyzed the relationship between changes in smoking behavior, relapse ideas, nicotine dependence, and willingness to quit among people trying to quit in Beijing community during the epidemic and their length of staying at home, psychological state, and smoking status. It provides a reference for further optimizing smoking cessation service during the period of regular epidemic prevention and control and improving strategies to guide smoking cessation behavior.

Among the 388 smokers trying to quit who participated in this telephone survey, 97 people stated that they had succeeded before the epidemic, and the quitting rate is $25.0 \%$. During the epidemic, 155 people stated that they had successfully quit, and the quitting rate reached $39.9 \%$, an increase of $14.9 \%$ compared with that before the epidemic. The average daily tobacco consumption of people trying to quit (7.10 \pm 8.473$)$ during the epidemic decreased slightly $(7.77 \pm 8.405$ before the epidemic),also. During the epidemic, the scores of the nicotine dependence assessment $(1.91 \pm 2.449)$ of those trying to quit were lower than that before the epidemic $(4.35 \pm 2.518)$, and the scores of the willingness to quit assessment during the epidemic $(38.17 \pm 12.377)$ were significantly higher than those before the epidemic $(19.78 \pm 9.103)$, which shows that during the epidemic, people were more positive in quitting smoking.

This reminds us that the environment of regular epidemic prevention and control may be very beneficial to better help smokers trying to quit and to establish a good tobacco control environment. During the epidemic prevention and control period, governments at all levels in China issued policies to strictly control gatherings, and many social events were not allowed to be held. At the same time, for many smokers, smoking behavior 
at home is supervised or stopped by their family members, and it is mandatory to wear masks outdoors, smoking behavior therefore becomes very inconvenient and unsafe. These measures are very important for tobacco control and quitting behavior promotion and have positive effects. In the future environment of regular epidemic prevention and control, we should seize the opportunity to take advantage of the favorable conditions provided by various epidemic prevention policies for tobacco control, strengthen tobacco control propaganda, encourage smokers to quit, and further enhance the effectiveness of quitting behaviors, thus contributing to the building of a smoke-free environment.

During the epidemic, each day, $74.7 \%$ of people trying to quit smoking stayed at home for more than $80 \%$ of the day, and even stayed at home all day. Although the epidemic is severe, they are relatively positive and optimistic in general, and stated that they are almost as usual or relatively calm. However, some subjects expressed some negative emotions, including anxiety, depression, panic, or suspicion. During the epidemic, the main reasons for smoking are smoking addiction or habit, entertainment, sorrow and boredom, and some people may also keep smoking because they want to refresh their minds, need to attend social and business activities, imitate out of curiosity, and relieve stress. For those who have not quit smoking, the change in tobacco consumption before and after the epidemic is mainly related to the generation of smoking thoughts, the psychological states of depression and calmness, relieve sorrow and boredom, addiction and smoking habits. Smoking thoughts are related to the psychological state of calmness and the need to relieve sorrow and boredom. The degree of nicotine dependence during the epidemic is related to the depressed psychological state and the willingness to quit smoking during the epidemic. The willingness to quit smoking is related to the daily length of staying at home and the degree of nicotine dependence during the epidemic. For tobacco-dependent patients who have successfully quit smoking, the change in daily tobacco consumption before and during the epidemic is related to the psychological state of depression, the need to relieve sorrow and boredom and relieve pressure, addiction and smoking habits, and willingness to quit smoking during the epidemic. The distribution difference of idea of relapse in the psychological state of depression, the need to relieve sorrow and boredom, entertain, and relieve pressure is statistically significant. The degree of nicotine dependence during the epidemic is related to the length of staying at home per day, calmness, and addiction and smoking habits. The willingness to quit smoking is related to the need to entertain, addiction and smoking habits,

The distribution difference of different length of staying at home in the tobacco consumption change and the idea of relapse in two groups of people trying to quit smoking is of no statistical significance, but is related to the changes in persistent smokers' willingness to quit smoking and ex-smokers' nicotine dependence during the epidemic. With the increase of time staying at home, persistent smokers tend to be more willing to quit smoking. The results of this study showed that during the epidemic, the willingness of both groups to quit smoking increased significantly compared with that before the epidemic. This possibly can be explained by the fact that the subjects spent most of their time at home with their family members, who supervised their smoking behavior and supported their quitting behavior, or the prohibition of smoking at home, had played a very positive role in enhancing the willingness to quit smoking among those trying to quit. Therefore, it is very necessary to create the environment of "smoke-free family", and family members should give quitters the greatest support and encouragement to help them quit. On the other hand, during the stay at home, the contact of people trying to quit smoking with their relatives and friends and other smoking peers decreased significantly, which means that social smoking behaviors were reduced. Quit smoking services such as drugs, regular follow-ups, and propaganda about tobacco hazards were also effective in gradually enhancing subjects' quitting awareness and willingness. Studies on smoking psychology of smokers show that social interaction is one of the main factors that affect smoking behaviors, and the need for social interaction leads to the motivation for smoking. In Chinese culture, tobacco is often used as a medium of interpersonal communication, and it is often regarded as a "respectful gift". "Delivering cigarettes" and "persuading others to smoke" are regarded as very common behaviors to show politeness, just like shaking hands. Studies have pointed out that quitters are prone to have a strong craving for cigarettes due to the induction of vision when their peers smoke. The rate of social smoking behaviors with friends in the group of successful quitters is significantly lower than that of failed quitters group. This also suggests that our smoking peers are inextricably associated with the occurrence of smoking behavior, and home isolation during the epidemic has weakened this relationship. Therefore, it is suggested that quit smoking services should help people trying to quit smoking establish a correct cognitive attitude to cope with social smoking behaviors, and provide personalized skills and methods to address social behaviors like "delivering cigarettes" and "persuading others to smoke" to help them become more determined in quitting and thus continue to maintain their non-smoking status.

In addition, with the increase of time staying at home, the desire and dependence of tobacco use by ex-smokers also tend to increase. This may be due to the large amount of free time at home during the epidemic, and the main reasons for the smoking behavior of ex-smokers have been to relieve sorrow and boredom, entertain, smoking addiction and habits. Some unsuccessful quitters also expressed that they are prone to smoke in the need of relieve sorrow and boredom. Studies have pointed out that smoking is an exploration of the temptation situation that occurs in a certain environmental temptation situation and is formed after long-term repeated strengthening of the habit of smoking cessation failure, such as unconsciously smoking at rest or after getting up in the morning. Quitting smoking causes this behavior to be terminated, but environmental temptation situations become a risk factor for inducing relapse. During the stay at home, in a specific environment with ample rest time, the risk factor of environmental temptation situation is magnified. It is recommended to adopt smoking cessation behavior therapy to break the connection between smoking behavior and certain activities of people trying to quit, divert attention to cultivate new interests, and finally master the alternative behavior of smoking, and establish a healthy lifestyle without smoking. 
In this study, depression and calmness are related to the smoking behavior and relapse thoughts of people trying to quit smoking. Among them, the smoking behavior of people trying to quit in depression may increase, and those who have quit smoking in depression are much easier to have the idea of relapse, but calmness brings positive changes, helping quitters rationally and calmly view the changes brought by independent isolation during the epidemic, and adjust quitters' smoking cessation status. During the telephone follow-ups, some quitters reported that they had negative psychological emotions such as anxiety and depression after the outbreak of epidemic, which led to the behavior of continuing to smoke. The study pointed out that sedation and enjoyment are the main factors affecting the smoking behavior of smokers. Sedation means that smokers often use smoking as a means to reduce negative emotions. For example, smokers in this study tend to smoke when they feel depressed; enjoyment means that many smokers regard smoking as a way to increase positive emotions, thinking that smoking is a comfortable, leisurely thing to do, and can improve mood and make their thinking quicker. When the smoker is depressed, the effect of smoking will be further expanded, which is caused by the erroneous understanding of the nicotine addiction mechanism after tobacco ingestion by quitters. Therefore, health education should be carried out for people trying to quit smoking, popularizing knowledge about tobacco addiction and publicizing the harm of tobacco. For patients who are trying to quit smoking and those who have successfully quit smoking, they should learn to use the correct emotion management methods and avoid adopting tobacco as a way to improve mood.

\section{Conclusion}

In summary, the period of normalization of epidemic prevention and control has created new opportunities and favorable conditions for optimizing quit smoking services, promoting smoking cessation, and tobacco control. During the epidemic, the social activities of people trying to quit smoking have been greatly reduced, and they have more time staying at home. It is recommended that family members provide more support and encouragement to them, help them enhance their willingness to quit, strengthen their determination and confidence in quitting, and supervise them to reduce smoking behaviors to facilitate the building of "smoke-free family". Under the epidemic environment, quitters may have negative emotions like depression. Quit smoking services should provide guidance on alleviating negative emotions to help quitters break existing connections between their smoking habits and certain situations, cultivate new interests, and succeed and continue to quit smoking so as to experience a healthy smoke-free life.

\section{Abbreviations}

1.FTND

Fagerstrom Test of Nicotine Dependence

2. PSM

Propensity Score Matching

3. NNM

Nearest neighbor matching

\section{Declarations}

Ethics approval and consent to participate: This study has been approved by the Medical Ethics Committee of Capital Medical University (Z2019SY007), and an informed consent form has been signed with the subjects before the investigation.

Trial registration: ChiCTR, ChiCTR1900024991. Registered 6 August 2019 - Retrospectively registered, http://www.chictr.org.cn/listbycreater.aspx

Consent for publication: The authors declare that they consent for publication.

Availability of data and materials: The datasets used and/or analysed during the current study available from the corresponding author on reasonable request.

Competing interest: The authors declare that they have no competing interests.

Funding: This research was supported by the Foundation of National Key R\&D Program of China (2017YFC1309400).

Authors' Contributions: QJ and XL conceived the study design, conceptualized the ideas, and supervised the whole analytical procedure. KQ helps telephone surveys and data collection. $\mathrm{HL}$ and $\mathrm{HM}$ contributed to field surveys and data collection. XB and XQ also provide technical support for translation of manuscripts. All authors have read and approved the manuscript.

Acknowledgments: We appreciate all investigators who participated in this study. We thank the staff from the 19 communities who cooperated with us in recruiting people to quit smoking, provided us with a venue to conduct our research, coordinated the timing of our research, and helped us compile information about the participants. We thank Tobacco Medicine and Tobacco Cessation Centre at the China-Japan Friendship Hospital for all the assistance and support during the research. QJ is very grateful to XL for the thesis supervision and constant encouragement. We also thank $\mathrm{HL}, \mathrm{HM}$ and $\mathrm{KQ}$, who helped with the preliminary survey and data review, and XB, XQ who helped in the translation work.

Page $17 / 19$ 


\section{References}

[1] Ma Kaixuan, Zhang Yide, Hou Tianya, et al. Investigation of physical and mental health in isolated people during the outbreak of novel coronavirus pneumonia. Chinese Journal of Clinical Medicine[J]. 2020,27(1):36-40.

[2] Li Chaoyang, Wang Bin. Psychological status and influencing factors in family isolation persons during outbreak of COVID-19. Henan Journal of Preventive Medicine[J].2020,31(9): 644-648,676.

[3] Liu Han, Jin Qianying, Ma Hanqiao, et al. Research on the behavior of attempting to quit smoking and its influencing factors among community smokers in Beijing. Chinese Journal of Health Management[J]. 2020,14(1):70-75.

[4] Ma Hanqiao, Liu Han, Jin Qianying, et al. Psychological Characteristics and Nicotine Dependence in Smokers in the Community. Chinese General Practice.[J]. 2020,23(15):1889-1894,1903.

[5] Yang Tingzhong, Chen Dingwan. Study on situations of Leading to smoking quitting Relapse. Chinese Mental Health Journal[J]. 2004,18(3):188-190,195.

[6] Juncheng Qian,Min Cai,Jun Gao,et al.Trends in smoking and quitting in China from 1993 to 2003: National Health Service Survey data[J].Bull World Health Organ,2010,88(10):769-776.

[7] JiangBin, He Yao, Zuo Fang, et al. Effectiveness of Varenicline with counseling programs on smoking cessation in a targeted clinical setting in China. Chinese Journal of Epidemiology [J]. 2014,035(012):1349-1353.

[8] Pan Jue, Jin Wenting, Wang Xiaodan, et al. Psychometric property of Chinese version of the Fagerstrom Test of Nicotine Dependence. International Journal of Respiration[J]. 2010,30(5):266-269.

[9] Shen Mengxuan. Intervention system for psychological crisis of home isolators based on "Internet +" during COVID-19. Chinese Journal of Medical Device [J]. 2020,33(17):17-18.

[10] Yang Liquan, Zhang Yanfeng, Xu Ying, et al. Investigation on psychological stress in fighting against corona virus disease 2019 among community residents. Chinese Nursing Research [J]. 2020,34(7):1140-1145.

[11] Reynolds DL,Garay JR,Deamond SL,et al.Understanding \compliance and psychological impact of the SARS quarantine experience[J].Epidemiol Infect,2008, 136(7):997-1007.

[12] LEVENTHAL A M.ZVOLENSKY M J.Anxiety,depression,and cigarette smoking:a transdiagnostic vulnerability framework to understanding emotion-smoking comorbidity[J]. Psychol Bull.2015,141(1):176-212】

[13] Ho LLK, Li WHC, Cheung AT, Xia W, Wang MP, Cheung DYT, Lam TH. Impact of COVID-19 on the Hong Kong Youth Quitline Service and Quitting Behaviors of Its Users[J]. Int J Environ Res Public Health. 2020 Nov 13;17(22):8397.

[14] Dai Zhen, Chen Yuanli, Chen Yonghong , et al. Effects of smoking behavior and socioeconomic factors on smoking psychology in smokers. Chinese Journal of Public Health [J].2014,30(11): 1382-1385.

[15] Chu Chengjing, Zhang Lan, Yang Yanchun. Social-psychological factors on smoking cessation failure among adult males. Chinese Journal of Public Health[J]. 2012, 28(1):7-9.

[16] Chu A,Jiang N,Glantz,SA.Transnational tobacco industry promotion of the cigarette gifting custom in China[J].Tobacco Control,2011,20(4):e3.

[17] Deng Linyuan, Fang Xiaoyi, Wu YANG, et al. Craving and Related Emotions Induced by Smoking Cues. Psychological Science[J]. 2009,32(4): 966-969.

[18] Wang Xiangdong, Wang Xilin, Ma Hong. Manual of Mental Health Rating Scales (Revised Version)[M]. Beijing: Chinese Journal of Mental Health.1999:360-363.

[19] Leventhal, Adam M.,Zvolensky, Michael J..Anxiety, Depression, and Cigarette Smoking: A Transdiagnostic Vulnerability Framework to Understanding Emotion-Smoking Comorbidity[J]. Psychological Bulletin,2015,141(1):176-212.

\section{Figures}




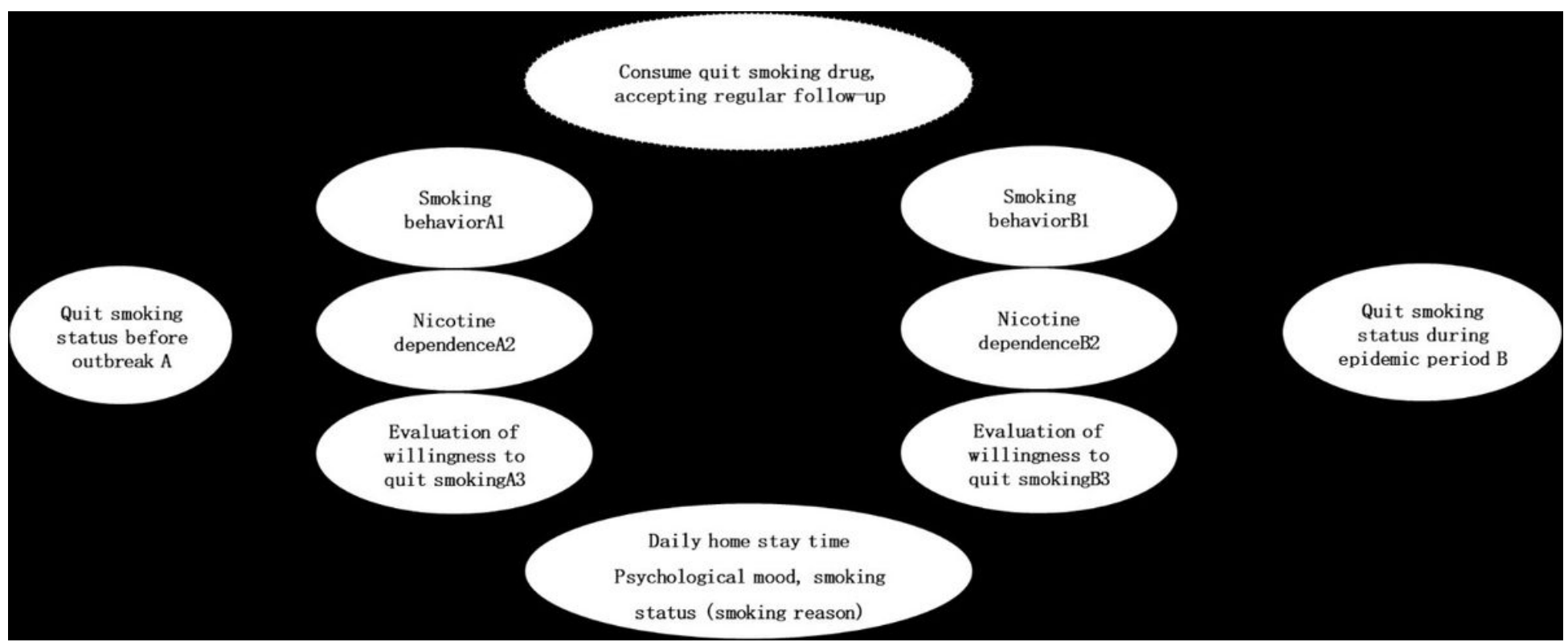

\section{Figure 1}

The subjects in this study had received three-month quit smoking services and six-month follow-up, which overlapped with the epidemic period. And again, use of quit smoking drugs and regular follow-ups might also was related to change in smoking behavior of people trying to quit smoking, see Figure 1. 